\title{
Flavour effects in gravitational leptogenesis
}

\author{
Rome Samanta $^{a}$ and Satyabrata Datta ${ }^{b}$ \\ ${ }^{a}$ Physics and Astronomy, University of Southampton, \\ Southampton, SO17 1BJ, U.K. \\ ${ }^{b}$ Saha Institute of Nuclear Physics, HBNI, \\ 1/AF Bidhannagar, Kolkata 700064, India \\ E-mail: romesamanta@gmail.com, satyabrata.datta@saha.ac.in
}

ABSTRACT: Within the Type-I seesaw mechanism, quantum effects of the right-handed $(\mathrm{RH})$ neutrinos in the gravitational background lead to an asymmetric propagation of lepton and anti-leptons which induces a Ricci scalar and neutrino Dirac-Yukawa coupling dependent chemical potential and therefore a lepton asymmetry in equilibrium. At high temperature, lepton number violating scattering processes try to maintain a dynamically generated lepton asymmetry in equilibrium. However, when the temperature drops down, the interactions become weaker, and the asymmetry freezes out. The frozen out asymmetry can act as a pre-existing asymmetry prior to the standard Fukugita-Yanagida leptogenesis phase ( $T_{i} \sim M_{i}$, where $M_{i}$ is the mass of $i$ th RH neutrino). It is then natural to consider the viability of gravitational leptogenesis for a given $\mathrm{RH}$ mass spectrum which is not consistent with successful leptogenesis from decays. Primary threat to this gravity-induced lepton asymmetry to be able to successfully reproduce the observed baryon-to-photon ratio is the lepton number violating washout processes at $T_{i} \sim M_{i}$. In a minimal seesaw set up with two $\mathrm{RH}$ neutrinos, these washout processes are strong enough to erase a pre-existing asymmetry of significant magnitude. We show that when effects of flavour on the washout processes are taken into account, the mechanism opens up the possibility of successful leptogenesis (gravitational) for a mass spectrum $M_{2} \gg 10^{9} \mathrm{GeV} \gg M_{1}$ with $M_{1} \gtrsim 6.3 \times 10^{6} \mathrm{GeV}$. We then briefly discuss how, in general, the mechanism leaves its imprints on the low energy $\mathrm{CP}$ phases and absolute light neutrino mass scale.

Keywords: Cosmology of Theories beyond the SM, CP violation, Neutrino Physics

ArXiv EPrint: 2007.11725 


\section{Contents}

1 Introduction 1

2 One flavour leptogenesis in two RH neutrino seesaw model 5

3 Flavour effect and successful gravitational leptogenesis 11

4 Parameter space and final results $\quad 17$

$\begin{array}{lll}5 & \text { Summary } & 21\end{array}$

\section{Introduction}

The dominance of matter over antimatter remains one of the outstanding questions in particle physics and cosmology. A simple and widely studied approach to this end is to create a lepton asymmetry and process it to the baryon asymmetry through $B-L$ conserving sphalerons $[1,2]$. The seesaw mechanism [3-7] which gives rise to the observed $\sim \mathrm{eV}$ scale [8] light neutrino masses also facilitates lepton number violating processes in the early universe. Within this mechanism, lepton number and CP-violating decays of heavy right-handed $(\mathrm{RH})$ Standard Model (SM) singlets when accompanied with out of equilibrium condition [9], create a lepton asymmetry (leptogenesis) [10-14] which is then converted to baryon asymmetry (baryogenesis/ matter-antimatter asymmetry) by sphaleron transition. Barring the SM gauge symmetry, in a most general scenario where the seesaw model is not subjected to any other symmetry (e.g., flavour symmetry [15-18]), it is natural to assume that the heavy $\mathrm{RH}$ states are hierarchical. It is then easy to show that the minimum RH mass scale pertaining to a successful leptogenesis is $M_{1} \sim 10^{9} \mathrm{GeV}$ (Davidson-Ibarra (DI) bound [19]) which is beyond the reach of the collider experiments. Obtaining testable predictions from leptogenesis thus requires either a lowering of the $\mathrm{RH}$ mass scale and going beyond the hierarchical limit or reduction in the number of free model parameters so that it can be tested indirectly in low energy neutrino experiments. To this end, whilst for a direct test, mechanisms such as leptogenesis from RH neutrino oscillation [20], a recently proposed mechanism of leptogenesis from Higgs decays [21] and resonant leptogenesis due to strongly quasi-degenerate heavy neutrinos $[11,22,23]$ are quite promising, for the latter, leptogenesis in grand unified theories like $\mathrm{SO}(10)$ [24-28] is worthwhile to give an emphasis on.

A different perspective in the leptogenesis scenario has also been introduced by considering the interplay of particle physics and gravity where the lepton asymmetry is not produced by the decays or oscillation of particles rather the asymmetry is sourced by gravitational interactions. For example, lepton asymmetry sourced by chiral Gravitational Waves 
(GW) [29-35] and by the interaction of lepton or baryon current with background gravity through a $\mathrm{C}$ and CP- violating operator $\partial_{\mu} R j^{\mu} / M^{2}$, where $R$ is the Ricci scalar [30, 36-44]. The lepton asymmetry from GW is a consequence of a chiral imbalance in the SM model which sources the asymmetry in the form of left- handed neutrinos, thus not easy to be realised in seesaw models [32]. On the other hand, the operator $\partial_{\mu} R j^{\mu} / M^{2}$ can be generated in seesaw models at two-loop level [45-48] (cf. figure 1) causing a chemical potential and hence a net lepton asymmetry in equilibrium proportional to the time derivative of $R$. The physical reason for the production of lepton asymmetry in this scenario could be attributed to the fact that $\mathrm{C}$ and $\mathrm{CP}$ violating operators when couple to the curvature at the quantum level, lead to asymmetric propagation (create a difference in lepton and anti-lepton self-energy) of matter and anti-matter-a phenomenon which is forbidden in flat space by translation and CPT invariance [45]. Starting from a minimally coupled Type-I seesaw Lagrangian

$$
-\mathcal{L}^{\text {seesaw }}=\sqrt{-g}\left[\bar{N}_{R i} \not D N_{R i}+f_{\alpha i} \bar{\ell}_{L \alpha} \tilde{H} N_{R i}+\frac{1}{2} \bar{N}_{R i}^{C}\left(M_{R}\right)_{i j} \delta_{i j} N_{R j}+\text { h.c. }\right],
$$

where $\sqrt{-g}$ is the square root of the metric determinant, $f$ is the neutrino Dirac-Yukawa coupling, $l_{L \alpha}=\left(\nu_{L \alpha} e_{L \alpha}\right)^{T}$ is the SM lepton doublet of flavour $\alpha, \tilde{H}=i \sigma^{2} H^{*}$ with $H=\left(H^{+} H^{0}\right)^{T}$ being the Higgs doublet and $M_{R}=\operatorname{diag}\left(M_{1}, M_{2}, M_{3}\right), M_{1,2,3}>0$, the generated equilibrium asymmetry at a temperature $T$ is given by [47]

$$
N_{B-L}^{e q}=\frac{\pi^{2} \dot{R}}{36(4 \pi)^{4}} \sum_{j>i} \frac{\operatorname{Im}\left[k_{i j}^{2}\right]}{\zeta(3) T M_{i} M_{j}}\left(\frac{M_{j}^{2}}{M_{i}^{2}}\right)^{p} \ln \left(\frac{M_{j}^{2}}{M_{i}^{2}}\right),
$$

where $k_{i j}=\left(f^{\dagger} f\right)_{i j}$ and $p=0,1[46,47]$. A dynamically generated asymmetry then freezes out once the relevant interactions (non-resonant relativistic $N_{i}$-exchange or $\Delta L=2$ processes) that try to maintain the asymmetry in equilibrium become weaker. Although for $p=1$, the equilibrium asymmetry and hence the frozen out asymmetry $\left(N_{B-L}^{G 0}\right)$ get enhanced hierarchically [47], in a generic seesaw model it is not trivial to realise a pure RH neutrino induced gravitational leptogenesis (or following ref. [48], Radiatively-induced gravitational leptogenesis (RIGL)). The reasons being, firstly, the gravitationally produced asymmetry competes with the asymmetry produced by RH neutrino decays, i.e., one has to distinguish the parameter space of each of the cases. On the other hand, even if by a suitable choice of $\mathrm{RH}$ mass spectrum one underestimates the contribution from $\mathrm{RH}$ neutrino decays towards successful leptogenesis, at the standard thermal leptogenesis phase $\left(T \sim M_{i}\right)$, the lepton number violating washout processes which are always present in a seesaw model in general wash out any pre-existing asymmetry $[49,50]$, here $N_{B-L}^{G 0}$, exponentially.

The latter one is a matter of concern in the present work. We stick to the minimal requirement of two RH neutrinos $\left(N_{1}\right.$ and $\left.N_{2}\right)$ [51-54] to generate light neutrino masses and choose the RH mass spectrum such that the decays from both the RH neutrinos $\left(N_{1,2^{-}}\right.$ leptogenesis) do not suffice to reproduce the correct baryon asymmetry. Thus we are left only with the asymmetry generated by the gravitational interaction of $\mathrm{RH}$ neutrinos, which 


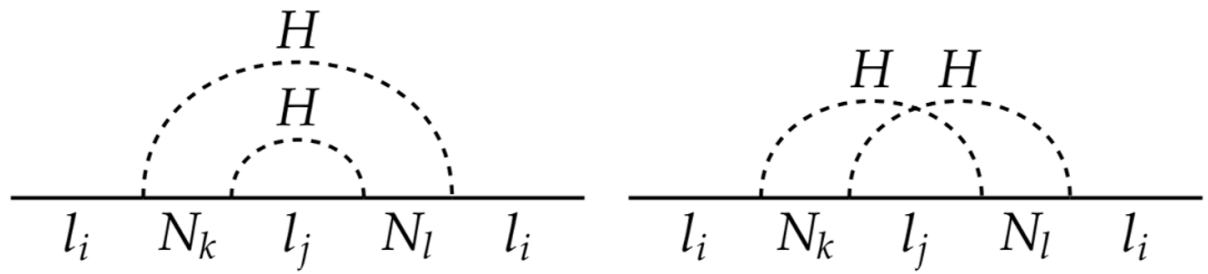

Figure 1. Two-loop diagrams in seesaw model that generate the $\partial_{\mu} R j^{\mu} / M^{2}$ operator, e.g., see ref. [47].

then faces washout by the lepton number violating $N_{i}$-interactions ( $N_{i}$-inverse decays). The final asymmetry can be represented mathematically as

$$
N_{B-L}^{G f} \sim N_{B-L}^{G 0} \mathcal{D}\left(\left[f^{\dagger} f\right]_{i i}\right)
$$

where $\mathcal{D}$ encodes an exponential dilution of the produced asymmetry by the washout processes. Strength of $\mathcal{D}$ then dictates the fate of successful gravitational leptogenesis. If $N_{B-L}^{G 0}$ is produced at a temperature $T_{0} \gg M_{i}$ (here $i=1,2$ ), it then faces ' $i$ ' number of washout at the scales $T_{i} \sim M_{i}$ by $N_{i}$-interactions $[55,56]$. In an unflavoured scenario (cf. eq. (1.3)), these washout effects are strong enough in minimal seesaw model (e.g., for a normal light neutrino mass ordering, $\mathcal{D}_{\min } \sim e^{-10^{3} m_{2} / \mathrm{eV}}, m_{2}$ being the lightest non-zero light neutrino mass and one has $m_{3}>m_{2}>m_{1}=0$.) to erase $N_{B-L}^{G 0}$. However, when effects of fast charged lepton interactions, i.e., interactions of lepton doublets with the RH component of charged leptons - popularly known as flavour effects in leptogenesis [57-63], on the washout processes are accounted for, one has to track the asymmetry in relevant flavours, and eq. (1.3) can be generalised to ${ }^{1}$

$$
N_{B-L}^{G f} \sim \sum_{\alpha} N_{B-L}^{G 0} \mathcal{D}\left(\left|f_{i \alpha}\right|^{2}\right) .
$$

Given the current neutrino oscillation data, we show that the strength of $\mathcal{D}\left(\left|f_{i \alpha}\right|^{2}\right)$ can be reduced drastically (e.g., dominantly in the electron flavour for normal light neutrino mass ordering) and consequently, $N_{B-L}^{G 0}$ does not face significant washout at $T_{i} \sim M_{i}$. This opens up the possibility to obtain pure gravitational leptogenesis in minimal seesaw models. Specifically, we consider two different hierarchical spectrum of RH masses, a) $10^{9} \mathrm{GeV} \ll M_{2} \ll 10^{12} \mathrm{GeV} \lesssim T_{0}, M_{1} \ll 10^{9} \mathrm{GeV}$, i.e., $M_{2}$ is in the two flavour regime and $M_{1}$ is in the three flavour regime (we shall explain flavour regimes in detail in section 3) b) $10^{12} \mathrm{GeV} \lesssim T_{0} \ll M_{2}, M_{1} \ll 10^{9} \mathrm{GeV}$, i.e., $M_{2}$ is in the unflavoured (one flavour) regime and $M_{1}$ is in the three flavour regime. For these spectrum of masses, it is well known that lepton asymmetry produced by RH neutrino decays is not adequate enough to be consistent with the observed baryon asymmetry, see e.g., [19, 64-66]. However, as mentioned earlier, $N_{B-L}^{G 0}$ which is produced gravitationally survives the washout effects owing to the fast charged lepton interactions which reduce the strength of the dilution

\footnotetext{
${ }^{1}$ Please note that, this mathematical form is naive and given only for the introduction purpose. In a realistic scenario, it requires more detailing which are discussed in relevant places.
} 


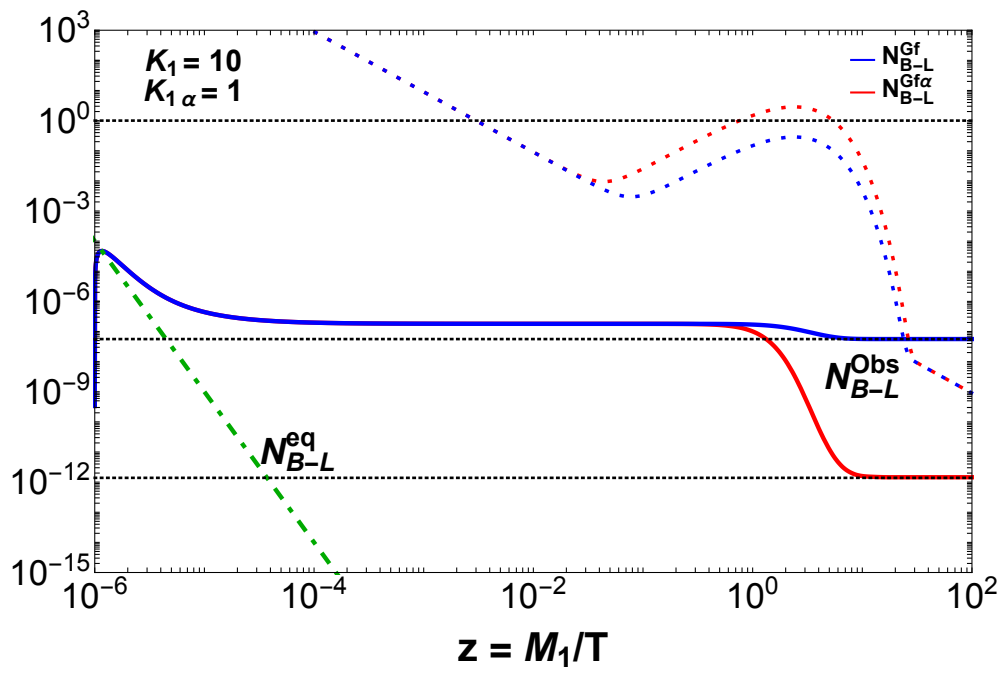

Figure 2. Evolution of Gravity induced lepton asymmetry for a benchmark value of $M_{1}=10^{7} \mathrm{GeV}$. The solid blue (red) line is the flavoured (unflavoured) asymmetry. The dashed blue (red) line is the flavoured (unflavoured) washout rate.

factor $\mathcal{D}$. After a detail quantitative study of flavour effects, we show that the spectrum b) with a normal light neutrino mass ordering (which is now favoured by neutrino oscillation data) facilitates successful gravitational leptogenesis and the lightest RH mass scale can be lowered to $\sim 6.3 \times 10^{6} \mathrm{GeV}$. Thus overall, our results have a two-fold impact on the studies related to leptogenesis in seesaw models. Firstly, possibility of successful leptogenesis with the lightest RH mass scale below DI bound on $M_{1}\left(M_{1} \gtrsim 10^{9} \mathrm{GeV}\right)$ [19]. Secondly, in minimal seesaw models, a new (non-standard) spectrum of $\mathrm{RH}$ masses emerge that reproduces correct baryon asymmetry. Of course, the production mechanism of the lepton asymmetry is now different - the asymmetry does not originate from the $\mathrm{RH}$ neutrino decays, rather it originates due to the quantum effects of $\mathrm{RH}$ neutrinos in a gravitational environment and the key difference is, that unlike the traditional leptogenesis scenario, here the production and washout occur in different flavour regimes. In a nutshell, entire discussion in this paper can be naively interpreted through figure 2 (which will be more detailed in section 3). As one sees, after the departure from the equilibrium the flavoured (blue) asymmetry does not encounter significant washout and explains the observed baryon asymmetry $\left(N_{B-L}^{\text {Obs }}\right)$ whereas the unflavoured (red) faces a strong washout at e.g., $T_{1} \sim M_{1}$ and fails to reproduce $N_{B-L}^{\mathrm{Obs}}$. In fact, we will see later that for the flavoured case, the dilution factor $D \sim e^{-K_{1 \alpha}}$ and there exists a large parameter space with $K_{1 \alpha} \ll 1$ so that practically there is no washout at $T_{1} \sim M_{1}$, however, in unflavoured case $D \sim e^{-K_{1}}$ and $K_{1} \gg 1$ thus the washout is strong. In either case, $K_{i(\alpha)}$ is called washout or decay parameter and is a function of $N_{i}$-Yukawa couplings.

The rest of the paper is organised as follows: In section 2, we discuss unflavoured leptogenesis for RH neutrino decays as well as for gravitational interaction. In section 3, we discuss flavoured leptogenesis scenario and show how in two RH neutrino seesaw model, 
a purely gravitational leptogenesis is realised. In section 4, we presented a detail numerical study and discussed the impact of RIGL mechanism on low energy neutrino observables. In section 5 we summarise our results.

\section{One flavour leptogenesis in two RH neutrino seesaw model}

In this section we briefly discuss leptogenesis from $\mathrm{RH}$ neutrino decays in the presence of a pre-existing lepton asymmetry created by the quantum effects of $\mathrm{RH}$ neutrinos in gravitational background. In a two RH neutrino seesaw model at a temperature $T_{B 1}<M_{1}$ where the $N_{1}$ interactions go out of equilibrium, the final asymmetry can be written as

$$
N_{B-L}^{f}=N_{B-L}^{G f}+N_{B-L}^{D f} .
$$

The number densities in eq. (2.1) are normalised to the co-moving number density of photons [12]. The first term is a contribution that originates due to the gravitational interactions of the RH neutrinos (after all the relevant washouts end) and the second term arises from the RH neutrino decays. Assuming the standard thermal history of the universe, the final baryon to photon ratio can be written as

$$
\eta_{B}=a_{\mathrm{sph}} \frac{N_{B-L}^{f}}{f_{\gamma}} \simeq 10^{-2} N_{B-L}^{f},
$$

where $f_{\gamma}$ is the photon dilution factor and $a_{\mathrm{sph}} \sim 1 / 3$ is the sphaleron conversion coefficient [12]. For a successful leptogenesis one has to compare eq. (2.2) to the observed value $\eta_{\mathrm{CMB}} \sim(6.3 \pm 0.3) \times 10^{-10}[67]$. First, we discuss the generation of the $B-L$ asymmetry $N_{B-L}^{D f}$ from RH neutrino decays. Starting from the neutrino mass terms in the seesaw Lagrangian in eq. (1.1)

$$
-\mathcal{L}_{\text {mass }}^{\nu, N}=\bar{\nu}_{L \alpha}\left(m_{D}\right)_{i \alpha} N_{R i}+\frac{1}{2} \bar{N}_{R i}^{C}\left(M_{R}\right)_{i j} \delta_{i j} N_{R j}+\text { h.c. }
$$

where $m_{D}=f v$ with $v=174 \mathrm{GeV}$ being the vacuum expectation value of the SM Higgs, the effective light neutrino mass matrix can be obtained with the seesaw mechanism [3] as

$$
M_{\nu}=-m_{D} M_{R}^{-1} m_{D}^{T} .
$$

The mass matrix in eq. (2.4) can be diagonalised by a unitary matrix $U$ as

$$
U^{\dagger} m_{D} M_{R}^{-1} m_{D}^{T} U^{*}=D_{m}
$$

where $D_{m}=-\operatorname{diag}\left(m_{1}, m_{2}, m_{3}\right)$ with $m_{1,2,3}$ being the physical light neutrino masses. We work in a basis where the $\mathrm{RH}$ neutrino mass matrix $M_{R}$ and charged lepton mass matrix $m_{\ell}$ are diagonal. Thus, the neutrino mixing matrix $U$ can be written as

$$
U=P_{\phi} U_{P M N S} \equiv P_{\phi}\left(\begin{array}{ccc}
c_{12} c_{13} & s_{12} c_{13} & s_{13} e^{-i \delta} \\
-s_{12} c_{23}-c_{12} s_{23} s_{13} e^{i \delta} & c_{12} c_{23}-s_{12} s_{13} s_{23} e^{i \delta} & c_{13} s_{23} \\
s_{12} s_{23}-c_{12} s_{13} c_{23} e^{i \delta} & -c_{12} s_{23}-s_{12} s_{13} c_{23} e^{i \delta} & c_{13} c_{23}
\end{array}\right) P_{M}
$$




\begin{tabular}{|c|c|c|c|c|c|}
\hline Parameter & $\begin{array}{c}\theta_{12} \\
\text { degrees }\end{array}$ & $\begin{array}{c}\theta_{23} \\
\text { degrees }\end{array}$ & $\begin{array}{c}\theta_{13} \\
\text { degrees }\end{array}$ & $\begin{array}{c}\Delta m_{21}^{2} \\
10^{-5}(\mathrm{eV})^{2}\end{array}$ & $\begin{array}{c}\left|\Delta m_{31}^{2}\right| \\
10^{-3}\left(\mathrm{eV}^{2}\right)\end{array}$ \\
\hline $3 \sigma$ ranges (NO) & $31.61-36.27$ & $41.1-51.3$ & $8.22-8.98$ & $6.79-8.01$ & $2.44-2.62$ \\
\hline $3 \sigma$ ranges (IO) & $31.61-36.27$ & $41.4-51.3$ & $8.26-9.02$ & $6.79-8.01$ & $2.42-2.60$ \\
\hline Best fit values (NO) & 33.82 & 48.6 & 8.60 & 7.39 & 2.53 \\
\hline Best fit values (IO) & 33.22 & 48.8 & 8.64 & 7.39 & 2.51 \\
\hline
\end{tabular}

Table 1. Input values used in the analysis (inclusive of SK data) [68].

where $P_{M}=\operatorname{diag}\left(e^{i \alpha_{M}}, 1, e^{i \beta_{M}}\right)$ is the Majorana phase matrix, $P_{\phi}=\operatorname{diag}\left(e^{i \phi_{1}}, e^{i \phi_{2}}, e^{i \phi_{3}}\right)$ is an unphysical diagonal phase matrix and $c_{i j} \equiv \cos \theta_{i j}, s_{i j} \equiv \sin \theta_{i j}$ with the mixing angles $\theta_{i j}=[0, \pi / 2]$. CP violation enters in eq. (2.6) through the Dirac phase $\delta$ and the Majorana phases $\alpha_{M}$ and $\beta_{M}$. It is convenient to parametrise (which can be straightforwardly derived from eq. (2.5)) the Dirac mass matrix as

$$
m_{D}=U \sqrt{D_{m}} \Omega \sqrt{M_{R}},
$$

where $\Omega$ is a $3 \times 3$ complex orthogonal matrix. As an aside, in table 1, let's present the latest fact file for the light neutrinos. Unlike the previously released data [69], present best-fit value $\left(\sim 221^{\circ}\right)$ for the Dirac CP violating phase $(\delta)$ exhibits a shift towards its $\mathrm{CP}$ conserving value for the Normal mass Ordering (NO), though for the Inverted mass Ordering (IO), best-fit of $\delta$ is still close to its maximal value $\left(\sim 282^{\circ}\right)$. The Majorana phases remain unconstrained and there is a preference of a Normal Ordering (NO) over an Inverted Ordering (IO).

Before the Electroweak Symmetry Breaking (EWSB), the RH neutrinos decays to lepton doublets and Higgs (cf. eq. (1.1)). The produced lepton doublets $\left|\ell_{i}\right\rangle$ can be written as a coherent superposition of the corresponding flavour states $\left|\ell_{\alpha}\right\rangle$ as,

$$
\begin{array}{ll}
\left|\ell_{i}\right\rangle=\mathcal{A}_{i \alpha}\left|\ell_{\alpha}\right\rangle & (i=1,2 ; \alpha=e, \mu, \tau) \\
\left|\bar{\ell}_{i}\right\rangle=\overline{\mathcal{A}}_{i \alpha}\left|\bar{\ell}_{\alpha}\right\rangle & (i=1,2 ; \alpha=e, \mu, \tau),
\end{array}
$$

where the tree-level amplitudes are given by

$$
\mathcal{A}_{i \alpha}^{0}=\frac{m_{D_{i \alpha}}}{\sqrt{\left(m_{D}^{\dagger} m_{D}\right)_{i i}}} \quad \text { and } \quad \overline{\mathcal{A}}_{i \alpha}^{0}=\frac{m_{D_{i \alpha}}^{*}}{\sqrt{\left(m_{D}^{\dagger} m_{D}\right)_{i i}}} .
$$

The asymmetry produced by the $\mathrm{CP}$-violating decays of the $\mathrm{RH}$ neutrinos is given by

$$
N_{B-L}^{D f}=\frac{3}{4} \sum_{i}^{2} \varepsilon_{i} \kappa_{i} .
$$

where $\varepsilon_{i}$ is the $\mathrm{CP}$ asymmetry parameter a nonzero value of which is ensured by the complex phases in the matrices $U$ and $\Omega$. The efficiency factor

$$
\kappa_{i}\left(z=M_{1} / T\right)=-\frac{4}{3} \int_{z_{\text {in }}}^{z} \frac{d N_{N_{i}}}{d z^{\prime}} e^{-\sum_{j} \int_{z^{\prime}}^{z} W_{j}\left(z^{\prime \prime}\right) d z^{\prime \prime}} d z^{\prime},
$$


contains the information of washout processes involving the inverse decays and lepton number violating scattering processes $[12,61]$. At a temperature $z_{B 1} \sim M_{1}$ the $N_{1}$-washout processes go out of equilibrium and in the hierarchical limit $M_{2} \gg M_{1}$ the efficiency factors for thermal initial abundance of the RH neutrinos can be computed as [12]

$$
\begin{aligned}
\kappa_{1}^{\infty} & =\frac{2}{K_{1} z_{B}\left(K_{1}\right)}\left(1-e^{-\frac{K_{1} z_{B}\left(K_{1}\right)}{2}}\right) \\
\kappa_{2}^{\infty} & =\frac{2}{K_{2} z_{B}\left(K_{2}\right)}\left(1-e^{-\frac{K_{2} z_{B}\left(K_{2}\right)}{2}}\right) e^{-\int_{0}^{\infty} W_{1}(z) d z}, \\
& \simeq \frac{2}{K_{2} z_{B}\left(K_{2}\right)}\left(1-e^{-\frac{K_{2} z_{B}\left(K_{2}\right)}{2}}\right) e^{-3 \pi K_{1} / 8}
\end{aligned}
$$

where

$$
z_{B}\left(K_{i}\right)=2+4 K_{i}^{0.13} e^{-\frac{2.5}{K_{i}}} \quad \text { and } \quad K_{i}=\frac{\left(m_{D}^{\dagger} m_{D}\right)_{i i}}{m^{*} M_{i}}
$$

with $m^{*} \simeq 10^{-3}$ being the equilibrium neutrino mass. The frozen out asymmetry $N_{B-L}^{D f}=$ $\sum_{i}^{2} \varepsilon_{i} \kappa_{i}^{\infty}$ then survives down to the low energy with the potential to explain the observed $\eta_{B}$. The flavoured CP asymmetry parameter is given by [11]

$$
\varepsilon_{i \alpha}=-\frac{1}{4 \pi v^{2} h_{i i}} \sum_{j \neq i}\left[\operatorname{Im}\left\{h_{i j}\left(m_{D}^{\dagger}\right)_{i \alpha}\left(m_{D}\right)_{\alpha j}\right\} g\left(x_{i j}\right)+\frac{\left(1-x_{i j}\right) \operatorname{Im}\left\{h_{j i}\left(m_{D}^{\dagger}\right)_{i \alpha}\left(m_{D}\right)_{\alpha j}\right\}}{\left(1-x_{i j}\right)^{2}+h_{j j}^{2}\left(16 \pi^{2} v^{4}\right)^{-1}}\right] \text {, }
$$

where $h_{i j}=\left(m_{D}^{\dagger} m_{D}\right)_{i j}, x_{i j}=M_{j}^{2} / M_{i}^{2}$ and $g\left(x_{i j}\right)$ is given by

$$
g\left(x_{i j}\right)=\left[\sqrt{x_{i j}}\left[1-\left(1+x_{i j}\right) \ln \left(\frac{1+x_{i j}}{x_{i j}}\right)\right]+\frac{\sqrt{x_{i j}}\left(1-x_{i j}\right)}{\left(1-x_{i j}\right)^{2}+h_{j j}^{2}\left(16 \pi^{2} v^{4}\right)^{-1}}\right] .
$$

Since $h_{i j}$ is a hermitian matrix, when summed over $\alpha$, the second term in eq. (2.16) vanishes. Using the orthogonal parametrisation for $m_{D}$ given in eq. (2.7), the total CP asymmetry parameter (which is relevant in one flavour approximation) can be written as

$$
\begin{aligned}
\varepsilon_{i} & =-\frac{1}{4 \pi v^{2}} \sum_{\alpha} \frac{\operatorname{Im}\left[M_{j} \sum_{k k^{\prime}} \sqrt{m_{k} m_{k^{\prime}}} m_{k} \Omega_{k i}^{*} \Omega_{k^{\prime} i}^{*} U_{k^{\prime} \alpha}^{\dagger} U_{\alpha k}\right] g\left(x_{i j}\right)}{\sum_{k^{\prime \prime}} m_{k^{\prime \prime}}\left|\Omega_{k^{\prime \prime} i}\right|^{2}} \\
& =-\frac{1}{4 \pi v^{2}} \frac{M_{j} g\left(x_{i j}\right) \sum_{k} m_{k}^{2} \operatorname{Im}\left[\Omega_{k i}^{*} \Omega_{k i}^{*}\right]}{\sum_{k^{\prime \prime}} m_{k^{\prime \prime}}\left|\Omega_{k^{\prime \prime} i}\right|^{2}}
\end{aligned}
$$

where $i, j(i \neq j)=1,2$. In the $N_{3}$ decoupling limit, the orthogonal matrices for NO $\left(m_{1}=0\right)$ and IO $\left(m_{3}=0\right)$ are given by

$$
\Omega^{\mathrm{NO}}=\left(\begin{array}{ccc}
0 & 0 & 1 \\
\cos \theta & \sin \theta & 0 \\
-\sin \theta & \cos \theta & 0
\end{array}\right), \quad \Omega^{\mathrm{IO}}=\left(\begin{array}{ccc}
\cos \theta & \sin \theta & 0 \\
-\sin \theta & \cos \theta & 0 \\
0 & 0 & 1
\end{array}\right)
$$


where $\theta=x+i y$ is a complex angle with $x$ and $y$ being real parameters. Using eq. (2.19) and maximising eq. (2.18) with respect to $x$ we get $^{2}$

$$
\begin{aligned}
& \varepsilon_{1}^{\mathrm{NO}}=-\frac{M_{2} g\left(x_{12}\right)}{4 \pi v^{2}}\left(m_{3}-m_{2}\right) \tanh 2 y, \\
& \varepsilon_{2}^{\mathrm{NO}}=\frac{M_{1} g\left(x_{21}\right)}{4 \pi v^{2}}\left(m_{3}-m_{2}\right) \tanh 2 y
\end{aligned}
$$

and

$$
\begin{aligned}
& \varepsilon_{1}^{\mathrm{IO}}=-\frac{M_{2} g\left(x_{12}\right)}{4 \pi v^{2}}\left(m_{2}-m_{1}\right) \tanh 2 y, \\
& \varepsilon_{2}^{\mathrm{IO}}=\frac{M_{1} g\left(x_{21}\right)}{4 \pi v^{2}}\left(m_{2}-m_{1}\right) \tanh 2 y .
\end{aligned}
$$

Before an explicit evaluation of the $\mathrm{CP}$ asymmetry parameters, we would like to emphasise on the following: The quantity

$$
\gamma_{i}=\sum_{j}\left|\Omega_{i j}^{2}\right| \geq 1
$$

accounts for the fractional contribution of the heavy $M_{j}$ states to a particular light neutrino $m_{i}$, and thus it can be treated as a measure of fine-tuning in the seesaw formula [70]. Since $\Omega$ belongs to $\mathrm{SO}(3, \mathbb{C})$, it is isomorphic to the Lorentz group and can be factorized as

$$
\Omega=\Omega^{\text {rotation }} \Omega^{\text {Boost }} .
$$

Using eq. (2.7) and eq. (2.10) one can derive a transformation relation between the states produced by the RH neutrinos $\left(\left|\ell_{j}\right\rangle\right)$ and the light neutrinos states $\left(\left|\tilde{\ell}_{i}\right\rangle\right)$ as

$$
\left|\ell_{j}\right\rangle=B_{j i}\left|\tilde{\ell}_{i}\right\rangle
$$

where the bridging matrix $B_{i j}$, first introduced in ref. [70] relates the heavy and the light states in general with a non-orthonormal transformation and is related to the orthogonal matrix as

$$
B_{j i}=\frac{\sqrt{m_{i}} \Omega_{j i}}{\sqrt{m_{k}\left|\Omega_{k j}\right|^{2}}} .
$$

For a choice of the orthogonal matrix $\Omega \equiv P$ (permutation matrix) which does not correspond to any fine-tuning (a particular heavy neutrino contributes to a particular light neutrino $[71,72])$, e.g.,

$$
\Omega^{\mathrm{NO}}=\left(\begin{array}{lll}
0 & 0 & 1 \\
1 & 0 & 0 \\
0 & 1 & 0
\end{array}\right)
$$

the heavy and the light states coincide as shown in figure 3. However for a general orthogonal matrix (cf. eq. (2.19)) which can be factorised as

$$
\Omega^{\mathrm{NO}}=\left(\begin{array}{ccc}
0 & 0 & 1 \\
\cos x & \sin x & 0 \\
-\sin x & \cos x & 0
\end{array}\right)\left(\begin{array}{ccc}
\cosh y & i \sinh y & 0 \\
-i \sinh y & \cosh y & 0 \\
0 & 0 & 1
\end{array}\right),
$$

\footnotetext{
${ }^{2}$ We choose $x=3 \pi / 4$ just for a demonstration purpose. However, the overall conclusion drawn is true for all values of $x$.
} 


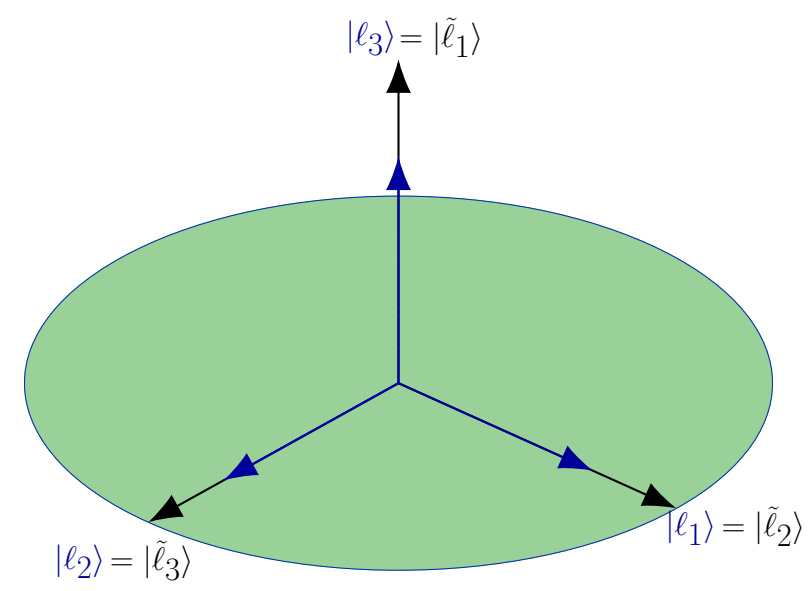

Figure 3. Pictorial representation of seesaw models with no fine-tuning, i.e., the states produced by the heavy neutrinos coincide with the orthonormal basis of the light neutrino states.

the orthonormality in the heavy states does not hold unless $x, y=0$. Due to the presence of the boost matrix, the heavy states are in general strongly non-orthonormal. Using eq. (2.24) the fine-tuning (boost) parameters can be calculated as

$$
\gamma_{2}=\gamma_{3} \equiv \gamma=\cosh 2 y
$$

Thus any non-zero value of $y$ will correspond to a certain level of fine-tuning in the seesaw formula. In fact, for one flavour leptogenesis at least this the case to obtain non-zero CP asymmetry (cf. eq. (2.20)). Coming back to the discussion of the CP asymmetry parameters, the function $g\left(x_{12}\right) \propto M_{1} / M_{2}$ and $M_{1} g\left(x_{21}\right) \propto M_{1}^{2} / M_{2}$. Now e.g., in eq. (2.20), taking $m_{3}-m_{2} \sim 0.1 \mathrm{eV}$, tanh $2 y \sim 1$ and $\kappa_{1}^{\infty} \sim 10^{-2}$ it is evident that one needs $M_{1} \sim 10^{9} \mathrm{GeV}$ to generate $N_{B-L} \sim 10^{-8}$. The contribution to the asymmetry from $N_{2}$ is negligible since the CP asymmetry parameter is suppressed by a factor $M_{1} / M_{2}$ and the efficiency factor $\kappa_{2}^{\infty}$ gets an exponential suppression by $N_{1}$-washout (cf. eq. (2.14)). Thus a two RH seesaw model with $M_{1}<10^{9} \mathrm{GeV}$ and $M_{2} \gg M_{1}$ does not lead to successful leptogenesis from RH neutrino decays. We now try to understand whether the asymmetry $N_{B-L}^{G 0}$ which is generated gravitationally leads to successful leptogenesis. Using the orthogonal parametrisation of $m_{D}$ in eq. (2.7), for $M_{2} \gg M_{1}$ the asymmetry $N_{B-L}^{G 0}$ in eq. (1.2) can be written as

$$
N_{B-L}^{e q}=\frac{\pi^{2} \dot{R}}{36(4 \pi v)^{4}} \frac{\sum_{k} m_{k}^{2} \operatorname{Im}\left[\Omega_{\mathrm{k} 1}^{*} \Omega_{\mathrm{k} 1}^{*}\right]}{\xi(3) T} \frac{M_{2}^{2}}{M_{1}^{2}} \ln \left(\frac{\mathrm{M}_{2}^{2}}{\mathrm{M}_{1}^{2}}\right)
$$

with $\dot{R}$ as

$$
\dot{R}=\sqrt{3} \sigma^{3 / 2}(1-3 \omega)(1+\omega) \frac{T^{6}}{M_{P l}^{3}},
$$

where $\sigma=\pi^{2} g^{*} / 30, M_{P l} \sim 2.4 \times 10^{18} \mathrm{GeV}$ and we opted for the $p=1$ solution for which the asymmetry gets enhanced hierarchically [47]. A non-zero value of $\dot{R}$ in radiation domination can be obtained by considering so called trace-anomaly in the gauge sector allowing $1-3 \omega \simeq 0.1$ [37]. In a seesaw model, the main obstacle to any pre-existing 

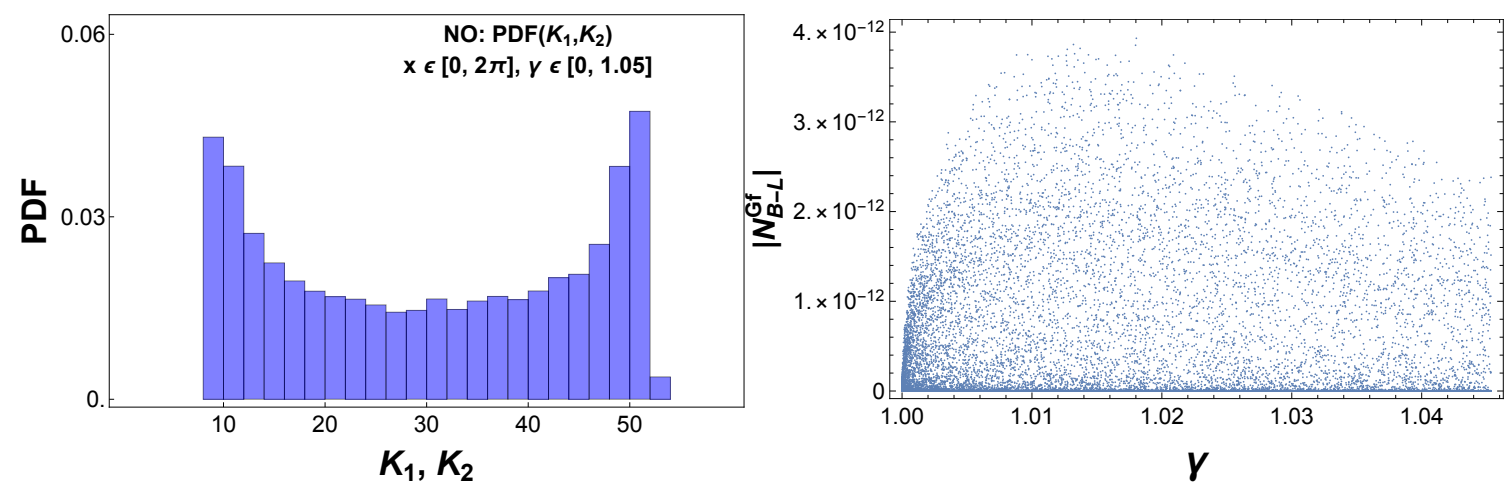

Figure 4. Left: Distribution of the total decay parameters $\left(K_{1,2}\right)$. Right: Magnitude of the gravitational asymmetry after $N_{1}$-washout.

asymmetry to survive down to the Electroweak scale is the washout processes involving lepton number violating $N_{i}$-interactions. First we consider $M_{2} \gg T_{0} \gg M_{1}$. Therefore, $N_{B-L}^{G 0}$ will face a washout at $T \sim M_{1}$ (by a factor $e^{-\frac{3 \pi}{8} K_{1}}$ ) $[55,56]$. The final asymmetry ${ }^{3}$ is then given by (cf. eq. (1.3))

$$
N_{B-L}^{G f}=\mathcal{E}_{\Delta L=2} N_{B-L}^{e q} \mathcal{D}\left(f_{i}\right)=N_{B-L}^{G 0} \mathcal{D}\left(f_{i}\right)=N_{B-L}^{G 0} e^{-\frac{3 \pi}{8} K_{1}},
$$

where the decay parameter $K_{i}$ can be expressed in terms of the orthogonal matrix as

$$
K_{i}=\frac{1}{m^{*}} \sum_{k} m_{k}\left|\Omega_{k i}\right|^{2}
$$

and $\mathcal{E}_{\Delta L=2}$ is the overall dilution factor due to the $\Delta L=2$ processes. In the left panel of figure 4 , we show a distribution of the decay parameters for minimally fine-tuned $(\simeq 5 \%)$ seesaw models by considering $x \in[0,2 \pi]$ and $\gamma \in[0,1.05]$. It is evident that due to the large values of the decay parameters the asymmetry $N_{B-L}^{G 0}$ gets washed out strongly and at $z_{B 1}$ one finds negligible value of $N_{B-L}^{G f}$ as shown in the right panel of figure 4 . The plot has been generated for a normal light neutrino mass ordering with a benchmark value of $M_{1}=10^{7} \mathrm{GeV}, M_{2}=10^{16} \mathrm{GeV}, \mathcal{E}_{\Delta L=2} \sim \mathcal{O}(1)$ (for a realistic flavour case we shall properly deal with $\mathcal{E}_{\Delta L=2}$ ) and considering the weak gravity condition $T \leq \sqrt{M_{1} M_{\mathrm{Pl}}}[48] .{ }^{4}$ This conclusion is true also for the strongly boosted seesaw systems $(\gamma \gg 1)$ as well as inverted mass ordering. The case of $T_{0} \gg M_{2} \gg M_{1}$ is more severe. In that case, due to a cascade washout $\left(\mathcal{D} \sim e^{-\left(K_{1}+K_{2}\right)}\right)$ at $T_{i} \sim M_{i}$, one obtains final asymmetry $\mathcal{O}\left(10^{-30}\right)$. Thus in the unflavoured regime, with the mass spectrum $M_{2} \gg M_{1}$, gravitational leptogenesis fails to explain the observed $\eta_{B}$. Whilst it is well known that for the discussed spectrum of masses,

\footnotetext{
${ }^{3}$ This expression is quite robust and perfectly reproduces numerical results as shown by the black dashed line in figure 2 , that matches the final value of the red solid line.

${ }^{4}$ In principle, there could be another condition, namely the low energy condition [48]: $z\left(=M_{1} / T\right) \geq$ $\left(10^{-2} M_{1} / M_{P l}\right)^{1 / 3}$ which will increase our chosen initial value of $z$ by an order of magnitude. Therefore, though we do sacrifice the strict validity of the effective Lagrangian, the point at which it becomes untrustworthy requires a more precise method of calculation. In any case, the gravitational effects go to zero in a dynamically controlled way for temperature well in excess of the validity of effective Lagrangian [73-75]. A more detail discussion in this issue can be found in [48].
} 
successful leptogenesis from decays is not possible even if one considers flavour effects (though the washouts get reduced, one simply does not have sufficient CP asymmetry from both the RH neutrinos) [64, 65], consideration of flavour effects in gravitational leptogenesis in this context requires investigation. This is the main objective of this work. The basic idea is that when one considers flavour effects, instead of total decay parameters $K_{i}$, flavoured decay parameters $K_{i \alpha}$ appear in the exponential washout (cf. eq. (2.33)). We will see in the next section that $K_{i \alpha}=P_{i \alpha} K_{i}$ with $P_{i \alpha}<1$ is the probability of a flavour state $\left|\ell_{\alpha}\right\rangle$ being in the state $\left|\ell_{i}\right\rangle$ associated to the heavy neutrinos. Thus $K_{i \alpha}$ is always weaker than $K_{i}$ and the washout effects get reduced which in turn enhance the probability for $N_{B-L}^{G f}$ being sizeable enough to be consistent with $\eta_{C M B}$.

\section{Flavour effect and successful gravitational leptogenesis}

Depending on the mass of the RH neutrinos, flavour effects play a crucial role in the computation related to leptogenesis. The one flavour regime (1FR) is typically characterised by $M_{i}>10^{12} \mathrm{GeV}$ where all the charged lepton flavours are out of equilibrium, and thus the lepton doublet $\left|\ell_{i}\right\rangle$ produced by the decay of the RH neutrinos or other external sources can be written as a coherent superposition of the corresponding flavour states $\left|\ell_{\alpha}\right\rangle$ as given in eq. (2.8) and eq. (2.9). Since there is hardly any interaction to break the coherence

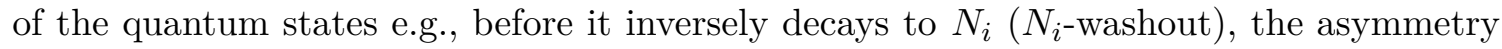
is produced along the direction of $\left|\ell_{i}\right\rangle$ (or $\left.\left|\bar{\ell}_{i}\right\rangle\right)$ in the flavour space. However, this is not the case when $M_{i}<10^{12} \mathrm{GeV}$, since below this scale, the $\tau$ charged lepton flavour comes into equilibrium and breaks the coherence of $\left|\ell_{i}\right\rangle$ states ( $\tau$ component gets measured [57]). Thus the relevant flavours that take part in the washout processes are the flavour $\tau$ and the coherent superposition of the flavours $e$ and $\mu$ - this is so called the two flavour regime (2FR) [57, 61]. Similarly when $M_{i}<10^{9} \mathrm{GeV}$, fast $\mu$ flavour interactions break the coherence of $e$ and $\mu$, therefore, one resolves all the three flavours (3FR). The flavour effects are taken into account by defining the branching ratios into individual flavours as $P_{i \alpha}=\left|\mathcal{A}_{i \alpha}\right|^{2}$ and $\bar{P}_{i \alpha}=\left|\overline{\mathcal{A}}_{i \alpha}\right|^{2}$. As a result, the decays (and hence the inverse decays, $\left.\Gamma_{i}^{\mathrm{ID}}=\Gamma_{i} \frac{N_{i}^{\mathrm{eq}}(z)}{N_{\ell}^{\mathrm{eq}}}\right)$ into individual flavours could be written as $\Gamma_{i \alpha} \equiv P_{i \alpha} \Gamma_{i}$ and $\bar{\Gamma}_{i \alpha} \equiv \bar{P}_{i \alpha} \bar{\Gamma}_{i}$ with $\sum_{\alpha}\left(P_{i \alpha}, \bar{P}_{i \alpha}\right)=1$. It is convenient also to introduce the flavoured decay parameter $K_{i \alpha}$ given by

$$
K_{i \alpha}=\frac{\Gamma_{i \alpha}+\bar{\Gamma}_{i \alpha}}{H\left(T=M_{i}\right)} \simeq \frac{P_{i \alpha}^{0}\left(\Gamma_{i}+\bar{\Gamma}_{i}\right)}{H\left(T=M_{i}\right)} \equiv P_{i \alpha}^{0} K_{i} \equiv \frac{\left|m_{D_{i \alpha}}\right|^{2}}{M_{i} m^{*}}
$$

which in terms of the orthogonal matrix can be re-expressed as

$$
K_{i \alpha}=\frac{1}{m^{*}}\left|\sum_{k} U_{\alpha k} \sqrt{m_{k}} \Omega_{k i}\right|^{2} .
$$

In the washout processes what matters is thus the flavoured decay parameters, e.g, the efficiency factor in eq. (2.12) can now be generalised to

$$
\kappa_{i \alpha}(z)=-\frac{4}{3} \int_{z_{\text {in }}}^{z} \frac{d N_{N_{i}}}{d z^{\prime}} e^{-\sum_{j} \int_{z^{\prime}}^{z} P_{j \alpha}^{0} W_{j}^{\mathrm{ID}}\left(z^{\prime \prime}\right) d z^{\prime \prime}} d z^{\prime}
$$


and the final value of any pre-existing asymmetry, e.g., $N_{B-L}^{G f}$ can be written ${ }^{5}$ as

$$
N_{B-L}^{G f}=\sum_{\alpha} p_{\alpha G} N_{B-L}^{G 0} e^{-\sum_{j} \int_{0}^{\infty} P_{j \alpha}^{0} W_{j}^{\mathrm{ID}}\left(z^{\prime \prime}\right) d z^{\prime \prime}} d z^{\prime}
$$

where $p_{\alpha G}$ is the probability for the $\left|\ell_{G}\right\rangle$ states being in the flavour $\alpha$. We assume production of gravitational asymmetry happens in the unflavoured regime (flavour blind) and conservatively consider $p_{\alpha G}=1 / 3$ through out [55]. As mentioned earlier, we intend to discuss the mass spectrum a) $10^{9} \mathrm{GeV} \ll M_{2} \ll 10^{12} \mathrm{GeV} \lesssim T_{0}$, and b) $10^{12} \mathrm{GeV} \lesssim T_{0} \ll M_{2}$, $M_{1} \ll 10^{9} \mathrm{GeV}$, therefore, eq. (3.3) is irrelevant in our discussion since this is the flavoured efficiency factors for lepton asymmetry produced by RH neutrino decays which for the chosen spectrum of RH masses, does not contribute significantly to the final $\eta_{B}$. Thus eq. (3.4) is the key equation for the entire analysis. As an aside, let's have a technical remark regarding the computation. We are considering strong flavour effect so that throughout the washout phases, charged lepton interactions dominate over the washout processes and the lepton system is completely incoherent in relevant flavour. Thus the formulae we use are the solutions of flavour diagonal Boltzmann Equations [56,61]. Otherwise, one has to solve exact density matrix equations which also take care of coherence among the flavour states. Strong flavour effect can be implemented by the condition

$$
W_{i}^{\max }\left(z_{i} \approx 1\right)<F_{\alpha}
$$

with $W_{i}$ is the washout parameter and $F_{\alpha}=\frac{\Gamma_{\alpha}}{H z_{i}}$ is the rate of charged lepton interaction. Taking $W_{i} \equiv W_{i}^{\mathrm{ID}}$ as $[12]$

$$
W_{i}^{\mathrm{ID}}=\frac{1}{4} K_{i} z_{i}^{2} \sqrt{1+\frac{\pi}{2} z_{i}} e^{-z_{i}}
$$

at $z_{i} \approx 1$ one arrives

$$
\frac{3 K_{i}}{20}<F_{\alpha}
$$

where $F_{\tau}=10^{12} / M_{i}$ and $F_{\mu}=10^{9} / M_{i}$. Eq. (3.7) when combined with eq. (2.34), translates into a condition on the boost parameter as

$$
\gamma \equiv \cosh 2 y \lesssim \frac{F_{\alpha}}{75}\left(\sum_{i} m_{i} / \mathrm{eV}\right)^{-1}
$$

This is the restriction one has to impose on the parameter space for the strong flavour effect to be strictly valid. Though in the end, we will see that eq. (3.8) has only a very mild effect on the parameter space pertaining to successful leptogenesis.

Having set up the basic formalism and technicalities of flavour effects in leptogenesis we now turn to the detailed analysis of the flavour effect in gravitational leptogenesis with the concerned spectrum of RH masses.

\footnotetext{
${ }^{5}$ We do not consider flavour effects on non-resonant $\Delta L=2$ processes since as we will see, bulk of the allowed solutions correspond to $\Delta L=2$ processes which are weaker and can be achieved in one flavour approximation. However in the case of precision calculation, it should be taken into account.
} 
$10^{9} \mathrm{GeV} \ll M_{2} \ll 10^{12} \mathrm{GeV} \lesssim T_{0}, M_{1} \ll 10^{9} \mathrm{GeV}$. In this case, $N_{2}$-washout happens in the two flavour regime and washout by $N_{1}$ acts in the three flavour regime. We can write $N_{B-L}^{G 0}\left(T \gg M_{2}\right)$ as a sum of two components:

$$
N_{B-L}^{G 0} \equiv N_{B-L}^{G 0 \tau}+N_{B-L}^{G 0 \tau^{\perp}}=p_{\tau G}^{0} N_{B-L}^{G 0}+\left(1-p_{\tau G}^{0}\right) N_{B-L}^{G 0},
$$

where $p_{\tau G}^{0}$ is the probability of $N_{B-L}^{G 0}$ being in the direction of $\tau$. Consequently, $1-p_{\tau G}^{0}$ is the probability in the $\tau^{\perp}$ direction.

Flavour coincidence. In this subsection, we present the basic idea of the flavour effects assuming $\tau$ and $\tau^{\perp}$ directions of $\left|\ell_{G}\right\rangle$ coincide with that of $\left|\ell_{2}\right\rangle$ (which is in general not true, see figure 5). There are now two stages of washout. The first one is at $T \sim M_{2}$ and the final one is at $T \sim M_{1}$. After the end of the first phase of washout, i.e., after $N_{2}$-interactions go out of equilibrium at $\left(T \sim z_{B 2}\right.$ ), the combined contribution of $\tau$ and $\tau_{G}^{\perp}$ component to the final asymmetry can be written as

$$
N_{B-L}^{G 1}=N_{B-L}^{G 1 \tau}+N_{B-L}^{G 1 \tau^{\perp}}=p_{\tau G}^{0} e^{-3 \pi\left(K_{2 \tau}\right) / 8} N_{B-L}^{G 0}+\left(1-p_{\tau G}^{0}\right) e^{-3 \pi\left(K_{2 e}+K_{2 \mu}\right) / 8} N_{B-L}^{G 0},
$$

where $N_{B-L}^{G i}$ is the frozen out asymmetry after $i$ th stage of washout. The frozen out asymmetries in each of the components will then face $N_{1}$-washout and at $z_{B 1}$ the components of final unwashed asymmetry can be written as

$$
\begin{aligned}
& N_{B-L}^{G 2 \tau}=N_{B-L}^{G 1 \tau} e^{-3 \pi\left(K_{1 \tau}\right) / 8}=p_{\tau G}^{0} e^{-3 \pi\left(K_{1 \tau}+K_{2 \tau}\right) / 8} N_{B-L}^{G 0} \\
& N_{B-L}^{G 2 \mu}=p_{\mu \tau_{2}^{\perp}}^{0} N_{B-L}^{G 1 \tau^{\perp}} e^{-3 \pi\left(K_{1 \mu}\right) / 8}=p_{\mu \tau_{2}^{\perp}}^{0}\left(1-p_{\tau G}^{0}\right) e^{-3 \pi\left(K_{2 e}+K_{2 \mu}+K_{1 \mu}\right) / 8} N_{B-L}^{G 0} \\
& N_{B-L}^{G 2 e}=p_{e \tau_{2}^{\perp}}^{0} N_{B-L}^{G 1 \tau^{\perp}} e^{-3 \pi\left(K_{1 e}\right) / 8}=p_{e \tau_{2}^{\perp}}^{0}\left(1-p_{\tau G}^{0}\right) e^{-3 \pi\left(K_{2 e}+K_{2 \mu}+K_{1 e}\right) / 8} N_{B-L}^{G 0}
\end{aligned}
$$

where the probabilities $p_{\alpha \tau_{2}^{\perp}}^{0}$ are given by

$$
p_{\alpha \tau_{2}^{\perp}}^{0}=\frac{p_{2 \alpha}^{0}}{\sum_{\alpha} p_{2 \alpha}^{0}}=\frac{K_{2 \alpha}^{0}}{\sum_{\alpha} K_{2 \alpha}^{0}} \text { with } \alpha=e, \mu .
$$

The final asymmetry is then given by

$$
N_{B-L}^{G f}=\sum_{\alpha} N_{B-L}^{G 2 \alpha} \text { with } \alpha=e, \mu, \tau .
$$

Projection dominance. In the previous discussion to have an overall idea of the washout processes we assume the states $\left|\ell_{\tau_{G}^{\perp}}\right\rangle$ and $\left|\ell_{\tau_{2}^{\perp}}\right\rangle$ in the $e-\mu$ plane share a common direction, i.e., $p_{G \tau_{2}^{\perp}} \equiv\left|\left\langle\ell_{\tau_{G}^{\perp}} \mid \ell_{\tau_{2}^{\perp}}\right\rangle\right|^{2}=1$. However, this is in general not true. Assuming flavour blind production of gravitational asymmetry, the probability $p_{G \tau_{2}^{\perp}}$ can be calculated as

$$
p_{G \tau_{2}^{\perp}}=\frac{1}{2} \frac{K_{2}}{K_{2 \tau^{\perp}}} \frac{\left|\sum_{k} \sqrt{m_{k}} U_{e k}^{*} \Omega_{k 2}^{*}+\sum_{k} \sqrt{m_{k}} U_{\mu k}^{*} \Omega_{k 2}^{*}\right|^{2}}{\sum_{k} m_{k}\left|\Omega_{k 2}\right|^{2}} .
$$

In figure 5 we plot a distribution of $p_{G \tau_{2}^{\perp}}$ with the $\gamma$. It is evident that $p_{G \tau_{2}^{\perp}}$ can have any values ranging from $0-1$. In fact, the most probable values are clustered around 

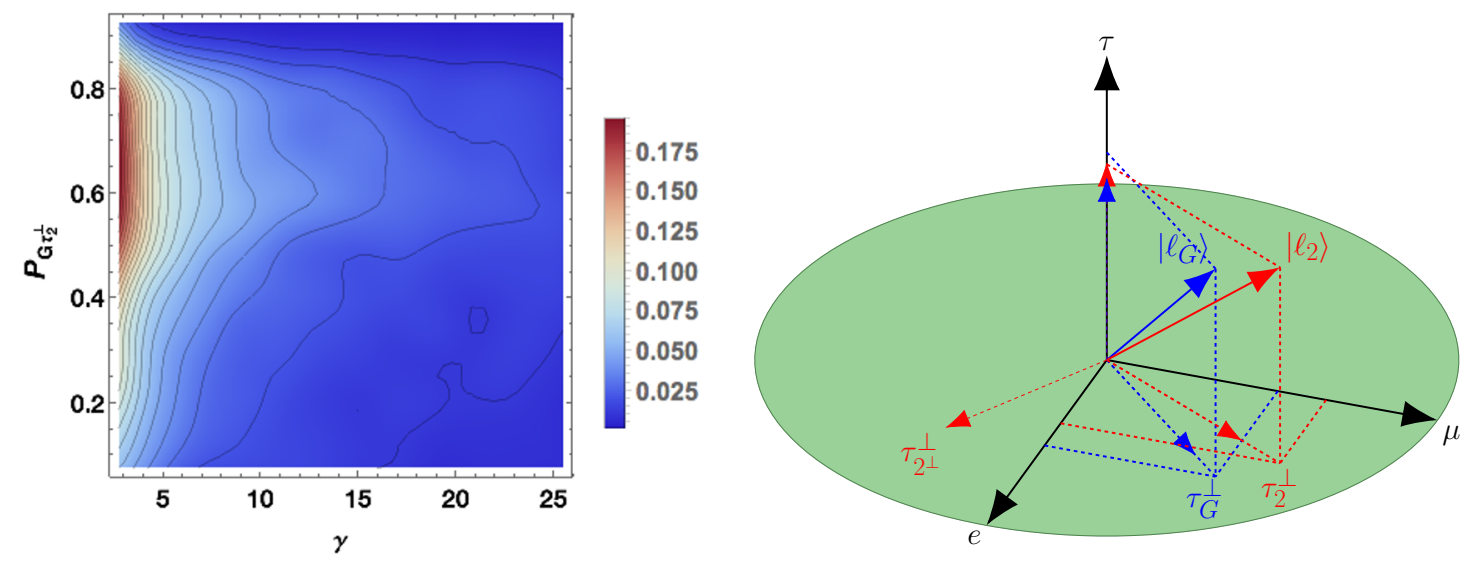

Figure 5. Left: Distribution of the overlap probability $p_{G \tau_{2}^{\perp}}$. Right: A visual representation of relevant flavour directions pertinent to leptogenesis for the mass spectrum $10^{9} \mathrm{GeV} \ll M_{2} \ll 10^{12}$ $\mathrm{GeV}, M_{1} \ll 10^{9} \mathrm{GeV}$.

$p_{G \tau_{2}^{\perp}} \simeq 0.7$. Thus component of $N_{B-L}^{G 0 \tau^{\perp}}$ which is in the direction of $\left|\ell_{\tau_{2}^{\perp}}\right\rangle$ will be washed out by $N_{2}$ interactions but the component which is orthogonal to $\left|\ell_{\tau_{2}^{\perp}}\right\rangle$ will escape $N_{2^{-}}$ washout. This is so called the projection effect first introduced in ref. [76] and then studied in detail e.g., in refs. $[56,61]$. The final baryon asymmetry at $z_{B 2}$ can now be written as

$$
\begin{aligned}
N_{B-L}^{G 1 \tau} & =p_{\tau G}^{0} e^{-3 \pi\left(K_{2 \tau}\right) / 8} N_{B-L}^{G 0}, \\
N_{B-L}^{G 1 \tau^{\perp}} & =N_{B-L}^{G 1 \tau_{2}^{\perp}}+N_{B-L}^{G 1 \tau_{2 \perp}^{\perp}}
\end{aligned}
$$

where

$$
\begin{aligned}
N_{B-L}^{G 1 \tau_{2}^{\perp}} & =\left(1-p_{\tau G}^{0}\right) p_{G \tau_{2}^{\perp}} e^{-3 \pi\left(K_{2 e}+K_{2 \mu}\right) / 8} N_{B-L}^{G 0}, \\
N_{B-L}^{G 1 \tau_{2 \perp}^{\perp}} & =\left(1-p_{\tau G}^{0}\right)\left(1-p_{G \tau_{2}^{\perp}}\right) N_{B-L}^{G 0}
\end{aligned}
$$

Now proceeding in the same way as the flavour coincidence case, after the $N_{1}$-washout, the asymmetries in each flavour can be written as

$$
\begin{aligned}
& N_{B-L}^{G 2 e}=\left(1-p_{\tau G}^{0}\right) e^{-3 \pi\left(K_{1 e}\right) / 8}\left[p_{G \tau_{2}^{\perp}} p_{e \tau_{2}^{\perp}}^{0} e^{-3 \pi\left(K_{2 e}+K_{2 \mu}\right) / 8}+\left(1-p_{e \tau_{2}^{\perp}}^{0}\right)\left(1-p_{G \tau_{2}^{\perp}}\right)\right] N_{B-L}^{G 0}, \\
& N_{B-L}^{G 2 \mu}=\left(1-p_{\tau G}^{0}\right) e^{-3 \pi\left(K_{1 \mu}\right) / 8}\left[p_{G \tau_{2}^{\perp}} p_{\mu \tau_{2}^{\perp}}^{0} e^{-3 \pi\left(K_{2 e}+K_{2 \mu}\right) / 8}+\left(1-p_{\mu \tau_{2}^{\perp}}^{0}\right)\left(1-p_{G \tau_{2}^{\perp}}\right)\right] N_{B-L}^{G 0}, \\
& N_{B-L}^{G 2 \tau}=p_{\tau G}^{0} e^{-3 \pi\left(K_{2 \tau}+K_{2 \tau}\right) / 8} N_{B-L}^{G 0} .
\end{aligned}
$$

For $p_{G \tau_{2}^{\perp}}=1$ we recover the formulae for flavour asymmetries presented in the flavour coincidence case. Note that, the second term (appears due to projection effect) in the first two equations in eq. (3.21) are dominating since compare to the other terms they escape $N_{2}$-washout and face washout by $N_{1}$ only at the second stage. We shall present numerical analysis only for the projection dominance since this is a more complete scenario compared to the flavour coincidence case. 


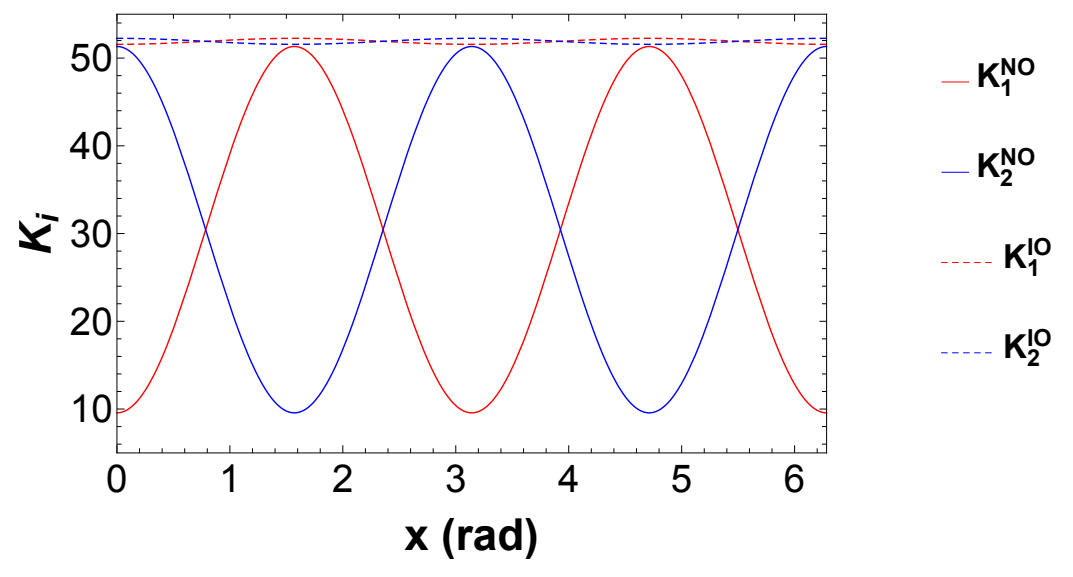

Figure 6. Unflavoured decay parameters in two RH neutrino seesaw models with $\gamma=1.05$.

b) $10^{12} \mathrm{GeV} \lesssim T_{0} \ll M_{2}, M_{1} \ll 10^{9} \mathrm{GeV}$ : In this case, $N_{2}$ does not participate in the washout process whereas the $N_{1}$-washout happens in the three flavour regime. The final asymmetry is then given by relatively simple formula

$$
N_{B-L}^{G f}=N_{B-L}^{G 0}\left(p_{e G} e^{-3 \pi\left(K_{1 e}\right) / 8}+p_{\mu G} e^{-3 \pi\left(K_{1 \mu}\right) / 8}+p_{\tau G} e^{-3 \pi\left(K_{1 \tau}\right) / 8}\right) .
$$

Given the expression for the final asymmetry in eq. (3.21) and eq. (3.22) we now try to understand quantitatively why in flavoured case the washout is less. First of all, using eq. (2.34), the unflavoured decay parameters can be written as

$$
\begin{aligned}
& K_{1}=\frac{1}{2 m^{*}}\left[\left(m_{\alpha}-m_{\beta}\right) \cos 2 x+\left(m_{\alpha}+m_{\beta}\right) \cosh 2 y\right], \\
& K_{2}=\frac{1}{2 m^{*}}\left[-\left(m_{\alpha}-m_{\beta}\right) \cos 2 x+\left(m_{\alpha}+m_{\beta}\right) \cosh 2 y\right],
\end{aligned}
$$

where for a normal ordering $\alpha=2, \beta=3$ and for an inverted ordering $\alpha=1, \beta=2$. Smaller values of the unflavoured decay parameters are obtained for $\gamma=\cosh 2 y \simeq 1$. For a normal light neutrino mass ordering, minimum value of $K_{1,2}^{\min }=m_{2} / m^{*} \equiv \sqrt{\Delta m_{12_{\min }}^{2}} / m^{*} \simeq$ 8.4 is obtained for $x=n \pi$ and $x=(2 n+1) \pi / 2$ respectively (cf. figure 6 ). On the other hand for an inverted mass ordering $K_{1,2}^{\min }=m_{1} / m^{*} \equiv \sqrt{\Delta m_{32_{\min }}^{2}-\Delta m_{12_{\min }^{2}}^{2}} / m^{*} \simeq 49$ is obtained for the same values of $x$. Therefore, in the unflavoured regime, washout $\left(\sim e^{-49}\right)$ in inverted mass ordering is more severe than the washout $\left(\sim e^{-8}\right)$ in normal mass ordering. However when flavour effect is included the scenario is quite different. The key physics that is responsible for weakening the strength of $K_{i \alpha}$ is the appearance of light neutrino mixing matrix $U$ in eq. (3.2). Using $3 \sigma$ neutrino oscillation data, in figure 7 we have shown a model independent triangle quantization of the flavour space. The upper panel corresponds to a normal and the lower panel corresponds to an inverted light neutrino mass ordering. One observes that for normal mass ordering the probability for $K_{i e}=P_{i e} K_{i}<1$ is much higher than the other two flavours. This means for the normal light neutrino ordering final asymmetry will be dominated by electron flavour (less washout in the electron flavour). On the other hand for inverted mass ordering the probability of having lower values $K_{i \alpha}$ 

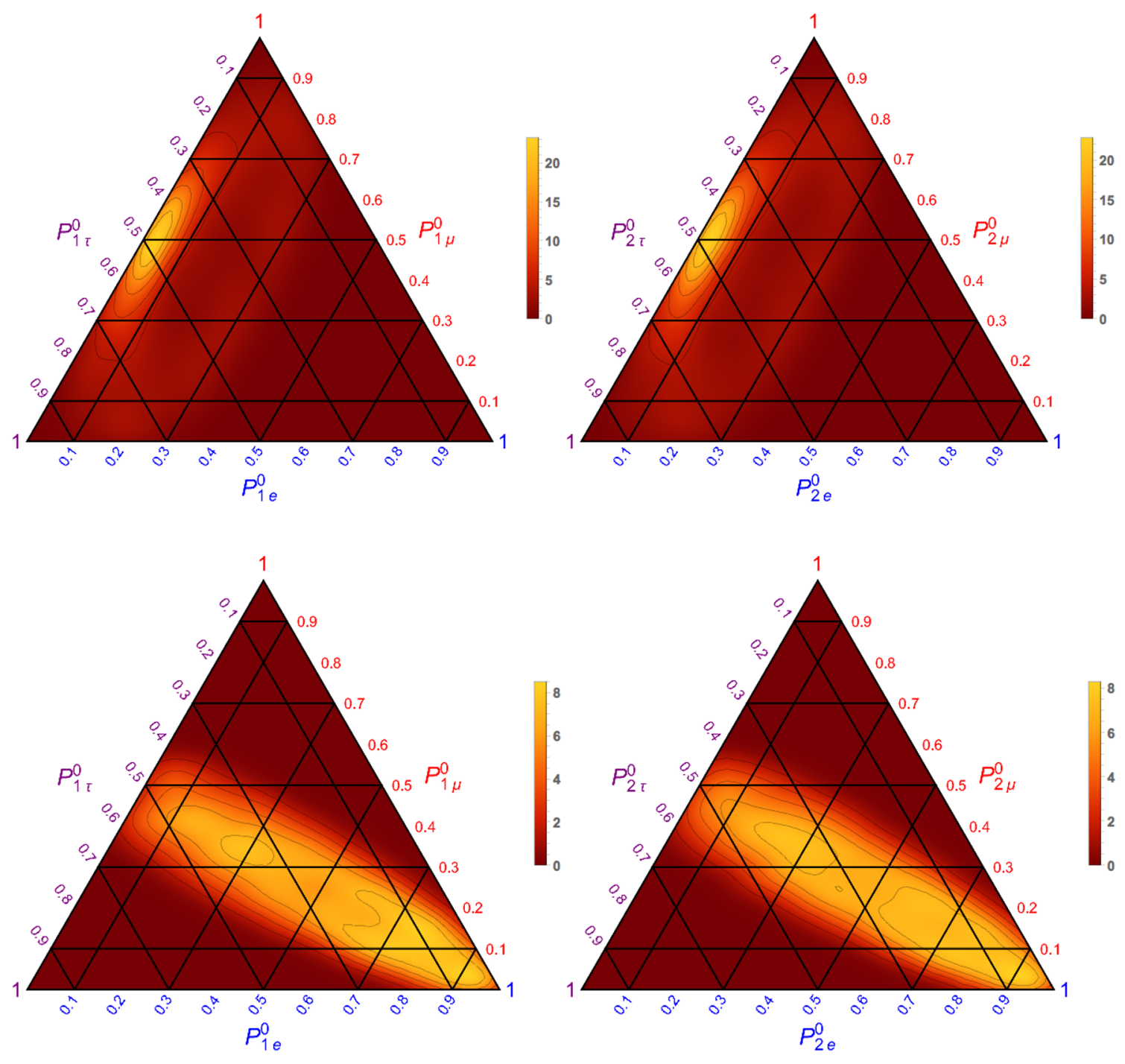

Figure 7. Ternary plots of the flavour probabilities. Upper panel: Normal mass ordering. Lower panel: Inverted mass ordering.

is quite democratic (though there is a bias towards $\mu$ and $\tau$ flavours). In any case, the overall information what we obtain from figure 7 is that we can have $K_{i \alpha} \ll 1$ to consider the washout at $T_{i} \sim M_{i}$ less significant. Now the leftover task is to precisely compute $N_{B-L}^{G 0}$ considering the effect of $\Delta L=2$ processes which tend to maintain the asymmetry $N_{B-L}^{e q}$ in equilibrium and therefore a dilution of the asymmetry between $z_{\text {in }}$ to $z_{0}$ (where the asymmetry freezes out). The frozen out asymmetry $N_{B-L}^{G 0}$ can be calculated by solving a simple Boltzmann equation $[47]^{6}$

$$
\frac{d N_{B-L}}{d z}=-W_{\Delta L=2}\left(N_{B-L}-N_{B-L}^{e q}\right)
$$

\footnotetext{
${ }^{6}$ Recently another term in the B.E has been introduced in ref. [48] which moderates the behaviour of $N_{B-L}$ at ultra-high temperature. However, in this paper we neglect that term for simplicity.
} 
where $W_{\Delta L=2}$ encodes the effect of $\Delta L=2$ process involving non-resonant $N_{1}$-exchange and is given by $[48,49]$

$$
W_{\Delta L=2}(z \ll 1) \simeq \frac{12 m^{*} M_{1}}{\pi^{2} v^{2} z^{2}}\left(\left[\frac{\bar{m}}{m^{*}}\right]^{2}+K_{1}^{2}\right) \text { with } \bar{m}=\sqrt{\sum_{i} m_{i}^{2}} .
$$

Since we intend to scan the entire parameter space using $3 \sigma$ neutrino oscillation data, it is convenient to solve the BE in eq. (3.25) analytically. To this end, we re-write eq. (3.25) as

$$
\frac{d N_{B-L}}{d z}=-\frac{\alpha}{z^{2}}\left(N_{B-L}-\frac{\beta}{z^{5}}\right)
$$

where

$$
\alpha=\frac{12 m^{*} M_{1}}{\pi^{2} v^{2}}\left(\left[\frac{\bar{m}}{m^{*}}\right]^{2}+K_{1}^{2}\right), \beta=\frac{\sqrt{3} \sigma^{3 / 2} M_{1}^{5}}{M_{P l}^{3}}(1-3 \omega)(1+\omega) \mathcal{Y} .
$$

The parameter $\mathcal{Y}$ which encodes the $\mathrm{CP}$ violation in the theory is given by

$$
\mathcal{Y}=\frac{\pi^{2}}{36(4 \pi v)^{4}} \frac{\sum_{k} m_{k}^{2} \operatorname{Im}\left[\Omega_{\mathrm{k} 1}^{*} \Omega_{\mathrm{k} 1}^{*}\right]}{\xi(3)} \frac{M_{2}^{2}}{M_{1}^{2}} \ln \left(\frac{\mathrm{M}_{2}^{2}}{\mathrm{M}_{1}^{2}}\right) \text {. }
$$

Starting from a vanishing initial abundance of $N_{B-L}(z)$, for large values of $z$ we find the analytical solution for $N_{B-L}^{G 0}$ as

$$
N_{B-L}^{G 0}=\frac{120 \beta}{\alpha^{5}}\left[1-e^{-\alpha / z_{\text {in }}}\right]-\frac{\beta e^{-\alpha / z_{\text {in }}}}{\alpha^{5}}\left[\sum_{n=1}^{5} \frac{5 !}{n !}\left(\frac{\alpha}{z_{\text {in }}}\right)^{n}\right] .
$$

Eq. (3.30) is the key analytical formula for $N_{B-L}^{G 0}$ with a minimum value of $z_{\text {in }}^{\min }=$ $\sqrt{M_{1} / M_{P l}}$ that we use to scan the parameter space.

\section{Parameter space and final results}

First, we compare the final frozen out value of the asymmetry $N_{B-L}^{G 0}$ that is obtained from the analytical formula in eq. (3.30) with the numerical solutions of eq. (3.25). In figure 8 for a benchmark value of $z_{\text {in }}=10^{-6}$ and $\beta=10^{-35}$, we show the evolution of the asymmetry (not taking into account the washout at $T_{1} \sim M_{1}$ ) for different values of $\alpha$. The coloured lines are the numerical solutions, and the black dashed lines represent analytically obtained values of $N_{B-L}^{G 0}$ which perfectly match the numerical results. It's worth noticing that for large values of $\alpha$, the asymmetry closely tracks its equilibrium value and therefore suffers a late freeze-out which in turn reduces the magnitude of the final asymmetry. We shall see this feature in our final results as well. We can now convincingly use the formula in eq. (3.30) along with eq. (3.21) and eq. (3.22) to scan entire parameter space using $3 \sigma$ neutrino oscillation data. In figure 9 , we show our final result. The upper panel contains parameter spaces for normal mass ordering for both the cases (left: case-a, right: case-b) and the lower panel contains the parameter spaces for the inverted mass ordering with the relevant cases ordered in a similar manner as in the upper panel. To generate the figures we fix $M_{1}=10^{7} \mathrm{GeV}$ for all the cases and $M_{2}=10^{16} \mathrm{GeV}$ for case-b and $M_{2}=10^{12}\left(M_{2}^{\max }\right) \mathrm{GeV}$ 


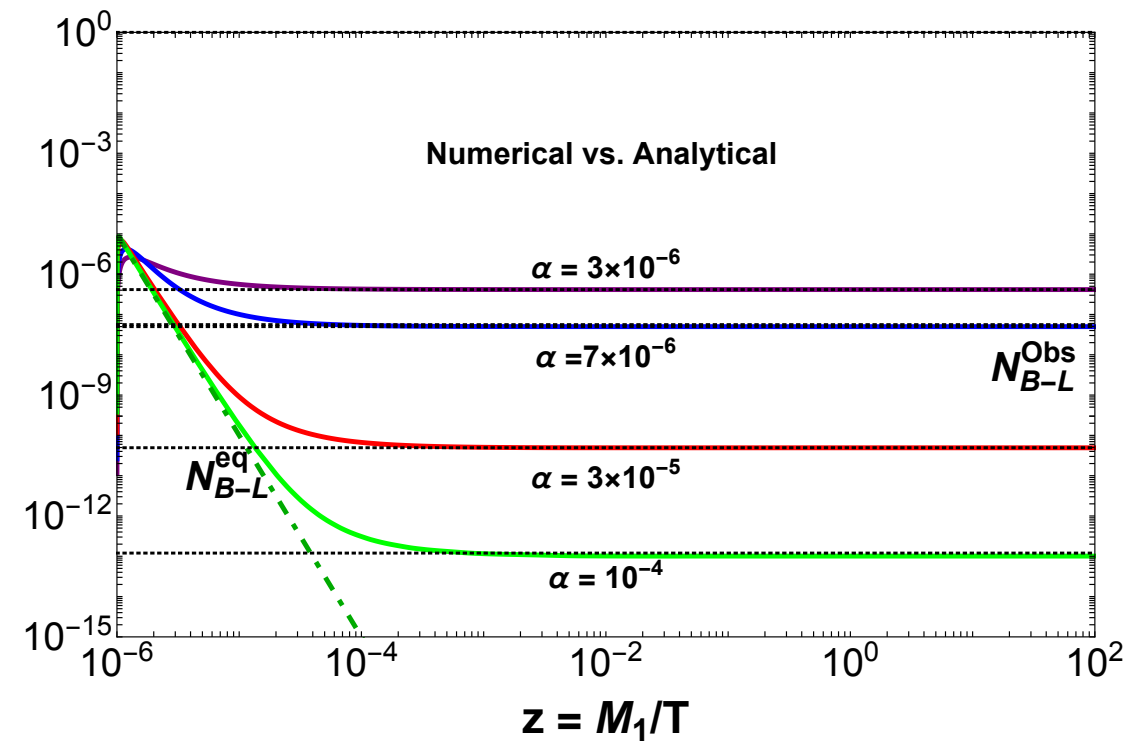

Figure 8. A numerical vs. analytical comparison of $N_{B-L}^{G 0}$. The coloured lines are numerical solutions and the black dashed lines are analytical yields.

for case-a (since we are considering $M_{2}$ to be in the two flavour regime for case-a). All the other parameters are randomly varied, i.e., the neutrino oscillation parameters are randomly generated following a Gaussian distribution, the parameters in the orthogonal matrix $x$ and $\gamma \equiv \cosh 2 y$ are varied within the interval $0-2 \pi$ and $0-50$ (as shown in the figures) with a flat distribution. In each of the cases, the magenta colour represents $N_{B-L}^{G 0}$ whereas the red, blue and green are the representative colours for electron, muon and tau flavour asymmetries. It is evident that both the light neutrino mass ordering in case-a are ruled out. In case-b, though the inverted ordering produces the $N_{B-L}^{G 0}$ within the correct range, the individual flavour asymmetries, as well as the sum of the flavour asymmetries, unfortunately, struggle to reproduce the correct asymmetry.

However, the normal mass ordering in case-b perfectly reproduces the observed asymmetry in all the flavours. An intriguing aspect is that the current neutrino oscillation data favour normal mass ordering and as we see, successful RIGL in minimalistic seesaw also requires normal mass ordering. We also note that the total asymmetry goes below the observed range for the value of $M_{1} \lesssim 6.3 \times 10^{6} \mathrm{GeV}$. In minimal seesaw, this can be regarded as the lower bound on $M_{1}$ for a successful RIGL. Note that now the bound is three orders of magnitude below the bound that is obtained in leptogenesis from RH neutrino decays $\left(M_{\text {Lightest }} \gtrsim 10^{9} \mathrm{GeV}\right)$. Coming into a bit more detail regarding the plots, for the case-a, as explained previously that the dominant contribution in the flavoured asymmetries comes from the projection effect for which the flavoured asymmetries escape $N_{2}$ washout (cf. eq. (3.21)). Despite a single-stage washout, the flavoured asymmetries struggle to produce large baryon asymmetry due to lack of hierarchical enhancement. However, unlike case-a, the hierarchical enhancement in case-b is much more stronger, and one obtains correct baryon asymmetry. Regarding the nature of the plots, firstly, as shown in figure 7 , for normal light neutrino ordering the strength of the washout (inverse decay) in 

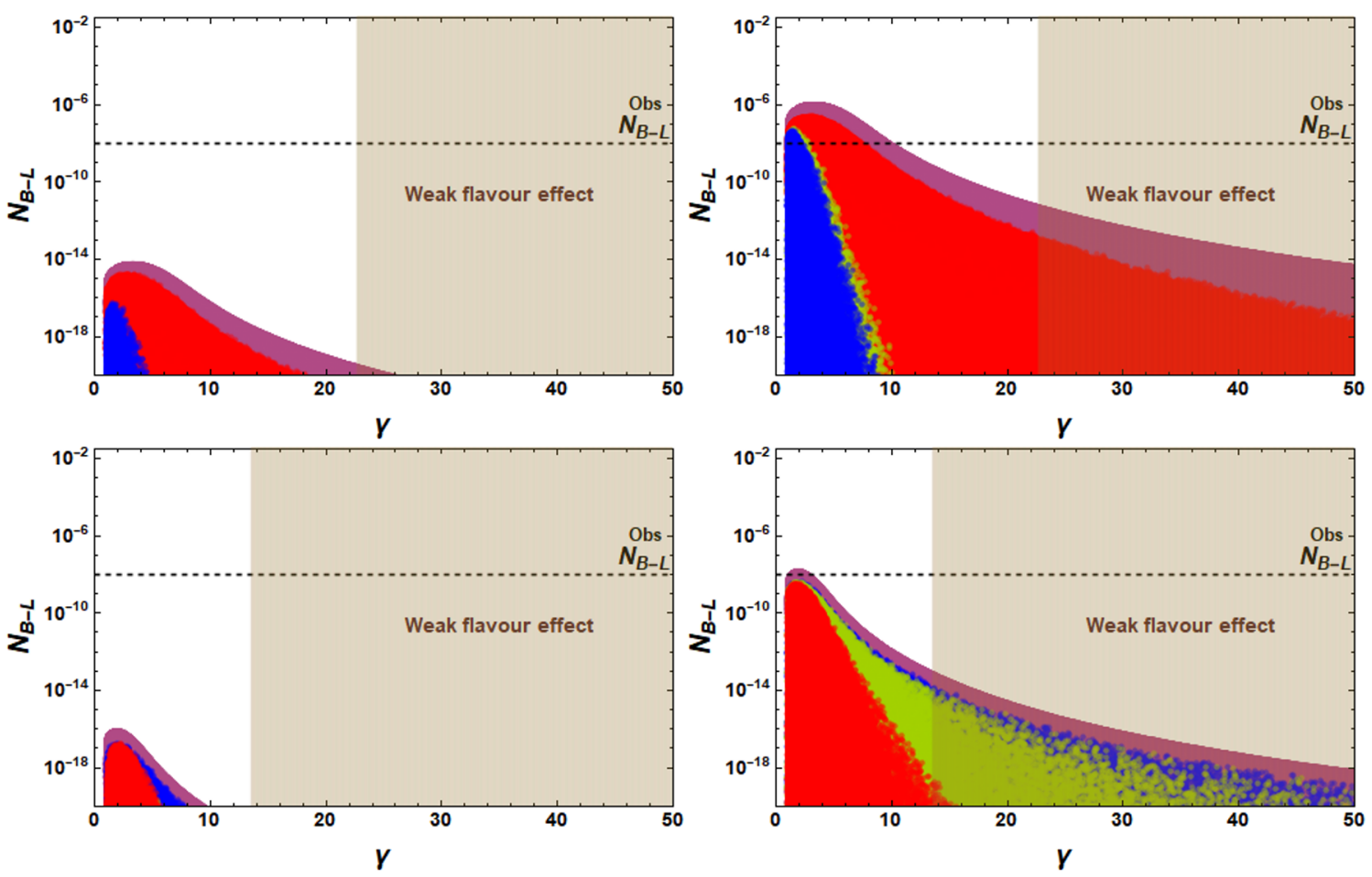

Figure 9. Upper panel left: case a) NO: $M_{2}=10^{12} \mathrm{GeV}, M_{1}=10^{7} \mathrm{GeV}$, right: case b) NO: $M_{2}=10^{16} \mathrm{GeV}, M_{1}=10^{7} \mathrm{GeV}$. Lower panel left: case a) IO: $M_{2}=10^{12} \mathrm{GeV}, M_{1}=10^{7} \mathrm{GeV}$, right: case b) IO: $M_{2}=10^{16} \mathrm{GeV}, M_{1}=10^{7} \mathrm{GeV}$. All the neutrino oscillation parameters are varied within $3 \sigma$ whereas $x$ and $\gamma$ are varied as $x \varepsilon[0,2 \pi]$ and $\gamma \varepsilon[0,50]$.

the electron flavour is much weaker than the other two flavours-making the RH neutrinos 'electrophobic'. This is clearly visible in the upper panel. In both the plots, the parameter space, as well as the magnitude of the asymmetry, is dominated by electron flavour (red region). Also notice that the parameter spaces of muon and tau flavours are more or less similar since washout in these flavours are of equal strength (cf. figure 7). However, for the inverted mass ordering the parameter space is dominated by the muon and tau flavour-a fact that was already anticipated in figure 7 (lower panel). Finally, the magnitude of the $N_{B-L}^{G 0}$ as well the flavoured asymmetries get reduced for large values $\gamma$. This is simply due to the fact that for large values of $\gamma$, the parameter $\alpha$ increases (since $K_{1}$ increases) which causes a late freeze-out of $N_{B-L}^{G 0}\left(z_{\text {in }}\right.$ and $z_{0}$ are well separated $\left.{ }^{7}\right)$ and hence a reduction in the magnitude of the final frozen out values of $N_{B-L}^{G 0}$. For large values of $\gamma$ the magnitude of the flavoured decay parameters increases as well. This is the reason that the flavour asymmetries are more suppressed for large values of $\gamma$. This we think an interesting aspect of RIGL - successful leptogenesis naturally requires less fine-tuning in seesaw formula. Note also that the condition of strong flavour effect is satisfied in the small- $\gamma$ region which

\footnotetext{
${ }^{7}$ This could be theoretically problematic for case-a since before the asymmetry freezes out $N_{2}$-washout starts to act and therefore eq. (3.18) is approximately valid. However, in any case, as we see, case-a lacks hierarchical enhancement and generates asymmetry several order of magnitude below $N_{B-L}^{\text {Obs }}$. Thus this consideration of this type hardly matters.
} 
is preferred by successful leptogenesis in RIGL. Before we conclude, we would like to make the following remarks (certainly not exhaustive):

- Should we wish to generalise this work into a three-RH neutrino scenario to probe a pure gravitational leptogenesis, we might consider two relevant hierarchical spectrum of masses I) $M_{2,3} \gg T_{\text {in }} \equiv T_{\mathrm{RH}}$ and $M_{1} \ll 10^{9} \mathrm{GeV}$ II) $M_{3} \gg T_{\text {in }} \equiv T_{\mathrm{RH}}$ and $M_{1,2} \ll 10^{9} \mathrm{GeV}$. In the former case, we expect similar results as in the present scenario (now the hierarchical enhancement is controlled by $M_{3} / M_{1}$ ). However, in the latter one we expect the lower bound on $M_{1}$ to be more stringent since the washout would be strong: $e^{-\left(K_{1 \alpha}+K_{2 \alpha}\right)}$ instead of $e^{-\left(K_{1 \alpha}\right)}$. However, to make a precise statement, this requires careful investigation.

- RIGL from low energy CP phases? As we see in eq. (2.31) that $N_{B-L}^{G 0}$ is free from the neutrino mixing matrix $U$ and thus in general RIGL is not directly connected to low energy CP phases. This is somewhat similar to nonthermal leptogenesis from inflaton decay [77]. However, there are well known techniques (models) to express the elements of $\Omega$ matrix in terms of low energy phases by reducing the number of parameters in seesaw models $[52,78]$. Keeping in mind significant progress in low energy neutrino experiments (neutrino parameters including the Dirac CP phase are getting measured with high statistical significance) models of these kinds are worth to explore in the light of RIGL which has never been done before.

- Imprints of RIGL on absolute neutrino mass scale and neutrino-less double beta decay? This point is a little bit tricky, and we have to opt for the paradigm of strong thermal leptogenesis $[55,79]$ where we don't want any pre-existing asymmetry to compete with the asymmetry that is produced by the decays. When $M_{2}$ is in the two flavour regime, and $M_{1}$ is in the three flavour regime, strong thermal leptogenesis can be successfully implemented with the following conditions:

$$
K_{2 \tau}, K_{1 e}, K_{1 \mu} \gg 1, K_{1 \tau} \ll 1
$$

By considering $K_{2 \tau} \gg 1$, we erase $\tau$ component of $N_{B-L}^{G 0}$ by washing it out. Note that $K_{2 \mu, 2 e} \gg 1$ do not help. Since in this case though with these conditions we can washout the asymmetry in the direction of $\tau_{2}^{\perp}(e+\mu)$, the asymmetry orthogonal to $\tau_{2}^{\perp}$ will survive and therefore one needs to wash it out at later stage by $N_{1}$ (washout effects act in all three directions of flavour) in the $e$ and $\mu$ flavours by choosing $K_{1 e}, K_{1 \mu} \gg 1$. Therefore, successful leptogenesis can be done by the decays of $N_{2}$ (One now introduces $N_{3}$ as well to have sufficient CP violation) in the $\tau$ flavour,

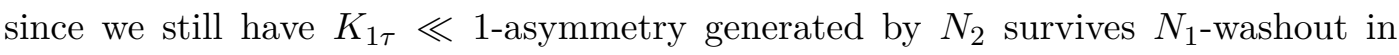
the direction of $\tau$. Such a hierarchical mass splitting can naturally be generated in $\mathrm{SO}(10)$ models [28], and the strong thermal conditions in eq. (4.1), in general, give lower bounds on $m_{1}$ and the neutrinoless double beta decay parameter $m_{e e}$ within the testable range of the cosmological and double beta decay experiments [79]. However, in all the previous studies, the magnitude of the pre-existing asymmetries have been put by hand, i.e., the magnitude does not depend on seesaw model parameters. 
But now, in this case, the most interesting part is, $N_{B-L}^{G 0}$ depends on the Yukawa couplings. Therefore, we expect the scenario would be more constrained than the previous studies. A dedicated analysis in this direction will be provided in a forthcoming publication [80].

- We have not discussed non-standard cosmological scenarios, e.g., a fast-expanding universe with an equation of state $1 / 3<\omega<1$ in which gravitational leptogenesis can be implemented even without hierarchical enhancement [48].

As a concluding remark, in seesaw models, RIGL mechanism opens up several interesting avenues which are worth exploring parallel to standard thermal leptogenesis from decays.

\section{Summary}

We discuss flavour effects on right-handed neutrino induced gravitational leptogenesis in the minimal seesaw model. We particularly consider two different spectrum of RH neutrino masses a) $10^{9} \mathrm{GeV} \ll M_{2} \ll 10^{12} \mathrm{GeV} \lesssim T_{0}, M_{1} \ll 10^{9} \mathrm{GeV}$, i.e., $M_{2}$ is in the two flavour regime and $M_{1}$ is in the three flavour regime b) $10^{12} \mathrm{GeV} \lesssim T_{0} \ll M_{2}, M_{1} \ll 10^{9}$ $\mathrm{GeV}$,.i.e., $M_{2}$ is in the unflavoured (one flavour) regime and $M_{1}$ is in the three flavour regime with $T_{0}$ being the temperature at which a frozen out lepton asymmetry is generated from gravitational mechanism. For these spectrum of masses, observed baryon asymmetry cannot be generated by RH neutrino decays. We show that for the same spectrum of masses, unflavoured gravitational leptogenesis does not successfully reproduce the observed baryon asymmetry. However, when flavour effects on washout processes are taken into account, for the case-b, the observed baryon asymmetry could be generated by the gravitational mechanism. We also show that the lower bound on $M_{1}$, in this case is, $\mathcal{O}\left(10^{6}\right) \mathrm{GeV}$ which is three orders of magnitude below than what is obtained from $\mathrm{RH}$ neutrino decays. We then discuss the future outlook of the gravitational leptogenesis mechanism, particularly its testability in low energy neutrino experiments.

\section{Acknowledgments}

The authors would like to thank Graham M. Shore for helpful discussions and useful correspondence of ref. [48] while we were finishing this draft. RS is supported by Newton International Fellowship (NIF 171202).

Open Access. This article is distributed under the terms of the Creative Commons Attribution License (CC-BY 4.0), which permits any use, distribution and reproduction in any medium, provided the original author(s) and source are credited.

\section{References}

[1] V.A. Kuzmin, V.A. Rubakov and M.E. Shaposhnikov, On the Anomalous Electroweak Baryon Number Nonconservation in the Early Universe, Phys. Lett. B 155 (1985) 36 [INSPIRE].

[2] E.W. Kolb and M.S. Turner, The Early Universe, Front. Phys. 69 (1990) 1 [InSPIRE]. 
[3] P. Minkowski, $\mu \rightarrow$ er at a Rate of One Out of $10^{9}$ Muon Decays?, Phys. Lett. B 67 (1977) 421 [INSPIRE].

[4] M. Gell-Mann, P. Ramond and R. Slansky, Complex Spinors and Unified Theories, Conf. Proc. C $\mathbf{7 9 0 9 2 7}$ (1979) 315 [arXiv: 1306.4669] [INSPIRE].

[5] T. Yanagida, Horizontal Symmetry and Masses of Neutrinos, Prog. Theor. Phys. 64 (1980) 1103 [INSPIRE].

[6] R.N. Mohapatra and G. Senjanović, Neutrino Mass and Spontaneous Parity Nonconservation, Phys. Rev. Lett. 44 (1980) 912 [INSPIRE].

[7] R.N. Mohapatra, Mechanism for Understanding Small Neutrino Mass in Superstring Theories, Phys. Rev. Lett. 56 (1986) 561 [InSPIRE].

[8] Particle Data Group collaboration, Review of Particle Physics, Phys. Rev. D 98 (2018) 030001 [INSPIRE].

[9] A.D. Sakharov, Violation of CP Invariance, $C$ asymmetry, and baryon asymmetry of the universe, Pisma Zh. Eksp. Teor. Fiz. 5 (1967) 32 [JETP Lett. 5 (1967) 24] [Sov. Phys. Usp. 34 (1991) 392] [Usp. Fiz. Nauk 161 (1991) 61] [InSPIRE].

[10] M. Fukugita and T. Yanagida, Baryogenesis Without Grand Unification, Phys. Lett. B 174 (1986) 45 [inSPIRE].

[11] A. Pilaftsis and T.E.J. Underwood, Resonant leptogenesis, Nucl. Phys. B 692 (2004) 303 [hep-ph/0309342] [INSPIRE].

[12] W. Buchmüller, P. Di Bari and M. Plümacher, Leptogenesis for pedestrians, Annals Phys. 315 (2005) 305 [hep-ph/0401240] [INSPIRE].

[13] S. Davidson, E. Nardi and Y. Nir, Leptogenesis, Phys. Rept. 466 (2008) 105 [arXiv:0802.2962] [INSPIRE].

[14] A. Riotto and M. Trodden, Recent progress in baryogenesis, Ann. Rev. Nucl. Part. Sci. 49 (1999) 35 [hep-ph/9901362] [INSPIRE].

[15] G. Altarelli and F. Feruglio, Discrete Flavor Symmetries and Models of Neutrino Mixing, Rev. Mod. Phys. 82 (2010) 2701 [arXiv: 1002.0211] [InSPIRE].

[16] H. Ishimori, T. Kobayashi, H. Ohki, Y. Shimizu, H. Okada and M. Tanimoto, Non-Abelian Discrete Symmetries in Particle Physics, Prog. Theor. Phys. Suppl. 183 (2010) 1 [arXiv: 1003.3552] [INSPIRE].

[17] S.F. King, Unified Models of Neutrinos, Flavour and CP-violation, Prog. Part. Nucl. Phys. 94 (2017) 217 [arXiv: 1701.04413] [inSPIRE].

[18] S.T. Petcov, Discrete Flavour Symmetries, Neutrino Mixing and Leptonic CP-violation, Eur. Phys. J. C 78 (2018) 709 [arXiv:1711.10806] [InSPIRE].

[19] S. Davidson and A. Ibarra, A lower bound on the right-handed neutrino mass from leptogenesis, Phys. Lett. B 535 (2002) 25 [hep-ph/0202239] [INSPIRE].

[20] E.K. Akhmedov, V.A. Rubakov and A. Smirnov, Baryogenesis via neutrino oscillations, Phys. Rev. Lett. 81 (1998) 1359 [hep-ph/9803255] [INSPIRE].

[21] T. Hambye and D. Teresi, Higgs doublet decay as the origin of the baryon asymmetry, Phys. Rev. Lett. 117 (2016) 091801 [arXiv: 1606.00017] [INSPIRE]. 
[22] P.S. Bhupal Dev, P. Millington, A. Pilaftsis and D. Teresi, Flavour Covariant Transport Equations: an Application to Resonant Leptogenesis, Nucl. Phys. B 886 (2014) 569 [arXiv: 1404.1003] [INSPIRE].

[23] P.S. Bhupal Dev, P. Millington, A. Pilaftsis and D. Teresi, Kadanoff-Baym approach to flavour mixing and oscillations in resonant leptogenesis, Nucl. Phys. B 891 (2015) 128 [arXiv: 1410.6434] [INSPIRE].

[24] P. Di Bari and A. Riotto, Successful type-I Leptogenesis with $\mathrm{SO}(10)$-inspired mass relations, Phys. Lett. B 671 (2009) 462 [arXiv:0809.2285] [InSPIRE].

[25] P. Di Bari and A. Riotto, Testing SO(10)-inspired leptogenesis with low energy neutrino experiments, JCAP 04 (2011) 037 [arXiv:1012.2343] [INSPIRE].

[26] C.S. Fong, D. Meloni, A. Meroni and E. Nardi, Leptogenesis in SO(10), JHEP 01 (2015) 111 [arXiv: 1412.4776] [INSPIRE].

[27] P. Di Bari, L. Marzola and M. Re Fiorentin, Decrypting SO(10)-inspired leptogenesis, Nucl. Phys. B 893 (2015) 122 [arXiv: 1411.5478] [INSPIRE].

[28] P. Di Bari and R. Samanta, The SO(10)-inspired leptogenesis timely opportunity, JHEP 08 (2020) 124 [arXiv : 2005. 03057] [inSPIRE].

[29] L. Álvarez-Gaumé and E. Witten, Gravitational Anomalies, Nucl. Phys. B 234 (1984) 269 [INSPIRE].

[30] S.H.-S. Alexander, M.E. Peskin and M.M. Sheikh-Jabbari, Leptogenesis from gravity waves in models of inflation, Phys. Rev. Lett. 96 (2006) 081301 [hep-th/0403069] [INSPIRE].

[31] R.R. Caldwell and C. Devulder, Axion Gauge Field Inflation and Gravitational Leptogenesis: A Lower Bound on B Modes from the Matter-Antimatter Asymmetry of the Universe, Phys. Rev. D 97 (2018) 023532 [arXiv: 1706.03765] [inSPIRE].

[32] P. Adshead, A.J. Long and E.I. Sfakianakis, Gravitational Leptogenesis, Reheating, and Models of Neutrino Mass, Phys. Rev. D 97 (2018) 043511 [arXiv:1711.04800] [INSPIRE].

[33] A. Papageorgiou and M. Peloso, Gravitational leptogenesis in Natural Inflation, JCAP 12 (2017) 007 [arXiv: 1708.08007] [INSPIRE].

[34] K. Kamada, J. Kume, Y. Yamada and J. Yokoyama, Gravitational leptogenesis with kination and gravitational reheating, JCAP 01 (2020) 016 [arXiv: 1911.02657] [INSPIRE].

[35] K. Kamada, J. Kume and Y. Yamada, Renormalization in gravitational leptogenesis with pseudo-scalar-tensor coupling, JCAP 10 (2020) 030 [arXiv:2007.08029] [INSPIRE].

[36] A.G. Cohen and D.B. Kaplan, Thermodynamic Generation of the Baryon Asymmetry, Phys. Lett. B 199 (1987) 251 [INSPIRE].

[37] H. Davoudiasl, R. Kitano, G.D. Kribs, H. Murayama and P.J. Steinhardt, Gravitational baryogenesis, Phys. Rev. Lett. 93 (2004) 201301 [hep-ph/0403019] [INSPIRE].

[38] H. Li, M.-z. Li and X.-m. Zhang, Gravitational leptogenesis and neutrino mass limit, Phys. Rev. D 70 (2004) 047302 [hep-ph/0403281] [INSPIRE].

[39] B. Feng, H. Li, M.-z. Li and X.-m. Zhang, Gravitational leptogenesis and its signatures in CMB, Phys. Lett. B 620 (2005) 27 [hep-ph/0406269] [INSPIRE].

[40] G. Lambiase and S. Mohanty, Gravitational Leptogenesis, JCAP 12 (2007) 008 [astro-ph/0611905] [INSPIRE]. 
[41] G. Lambiase and S. Mohanty, Leptogenesis by curvature coupling of heavy neutrinos, Phys. Rev. D 84 (2011) 023509 [arXiv: 1107.1213] [INSPIRE].

[42] G. Lambiase, S. Mohanty and A.R. Prasanna, Neutrino coupling to cosmological background: A review on gravitational Baryo/Leptogenesis, Int. J. Mod. Phys. D 22 (2013) 1330030 [arXiv: 1310.8459] [INSPIRE].

[43] J. Ellis, N.E. Mavromatos and S. Sarkar, Environmental CPT Violation in an Expanding Universe in String Theory, Phys. Lett. B 725 (2013) 407 [arXiv: 1304.5433] [INSPIRE].

[44] J.I. McDonald and G.M. Shore, Gravitational leptogenesis, C, CP and strong equivalence, JHEP 02 (2015) 076 [arXiv: 1411.3669] [INSPIRE].

[45] J.I. McDonald and G.M. Shore, Radiatively-induced gravitational leptogenesis, Phys. Lett. B 751 (2015) 469 [arXiv: 1508.04119] [INSPIRE].

[46] J.I. McDonald and G.M. Shore, Leptogenesis from loop effects in curved spacetime, JHEP 04 (2016) 030 [arXiv: 1512.02238] [INSPIRE].

[47] J.I. McDonald and G.M. Shore, Leptogenesis and gravity: baryon asymmetry without decays, Phys. Lett. B 766 (2017) 162 [arXiv:1604.08213] [inSPIRE].

[48] J.I. McDonald and G.M. Shore, Dynamical Evolution of Gravitational Leptogenesis, JHEP 10 (2020) 025 [arXiv:2006.09425] [INSPIRE].

[49] W. Buchmüller, P. Di Bari and M. Plümacher, Cosmic microwave background, matter-antimatter asymmetry and neutrino masses, Nucl. Phys. B 643 (2002) 367 [Erratum ibid. 793 (2008) 362] [hep-ph/0205349] [INSPIRE].

[50] W. Buchmüller, P. Di Bari and M. Plümacher, The neutrino mass window for baryogenesis, Nucl. Phys. B 665 (2003) 445 [hep-ph/0302092] [INSPIRE].

[51] S.F. King, Large mixing angle $M S W$ and atmospheric neutrinos from single right-handed neutrino dominance and U(1) family symmetry, Nucl. Phys. B 576 (2000) 85 [hep-ph/9912492] [INSPIRE].

[52] P.H. Frampton, S.L. Glashow and T. Yanagida, Cosmological sign of neutrino CP-violation, Phys. Lett. B 548 (2002) 119 [hep-ph/0208157] [INSPIRE].

[53] M. Raidal and A. Strumia, Predictions of the most minimal seesaw model, Phys. Lett. B $\mathbf{5 5 3}$ (2003) 72 [hep-ph/0210021] [INSPIRE].

[54] R. Sinha, R. Samanta and A. Ghosal, Generalized $\mathbb{Z}_{2} \times \mathbb{Z}_{2}$ in scaling neutrino Majorana mass matrix and baryogenesis via flavored leptogenesis, JHEP 12 (2017) 030 [arXiv: 1706.00946] [INSPIRE].

[55] E. Bertuzzo, P. Di Bari and L. Marzola, The problem of the initial conditions in flavoured leptogenesis and the tauon $N_{2}$-dominated scenario, Nucl. Phys. B 849 (2011) 521 [arXiv: 1007.1641] [INSPIRE].

[56] S. Blanchet, P. Di Bari, D.A. Jones and L. Marzola, Leptogenesis with heavy neutrino flavours: from density matrix to Boltzmann equations, JCAP 01 (2013) 041 [arXiv: 1112.4528] [INSPIRE].

[57] A. Abada, S. Davidson, A. Ibarra, F.-X. Josse-Michaux, M. Losada and A. Riotto, Flavour Matters in Leptogenesis, JHEP 09 (2006) 010 [hep-ph/0605281] [INSPIRE].

[58] E. Nardi, Y. Nir, E. Roulet and J. Racker, The importance of flavor in leptogenesis, JHEP 01 (2006) 164 [hep-ph/0601084] [INSPIRE]. 
[59] S. Blanchet and P. Di Bari, Flavor effects on leptogenesis predictions, JCAP 03 (2007) 018 [hep-ph/0607330] [INSPIRE].

[60] P.S.B. Dev, P. Di Bari, B. Garbrecht, S. Lavignac, P. Millington and D. Teresi, Flavor effects in leptogenesis, Int. J. Mod. Phys. A 33 (2018) 1842001 [arXiv:1711.02861] [InSPIRE].

[61] R. Samanta and M. Sen, Flavoured leptogenesis and CP ${ }^{\mu \tau}$ symmetry, JHEP 01 (2020) 193 [arXiv: 1908.08126] [INSPIRE].

[62] R. Samanta, M. Chakraborty, P. Roy and A. Ghosal, Baryon asymmetry via leptogenesis in a neutrino mass model with complex scaling, JCAP 03 (2017) 025 [arXiv:1610.10081] [INSPIRE].

[63] R. Samanta, R. Sinha and A. Ghosal, Importance of generalized $\mu \tau$ symmetry and its $C P$ extension on neutrino mixing and leptogenesis, JHEP 10 (2019) 057 [arXiv:1805.10031] [INSPIRE].

[64] S. Antusch, P. Di Bari, D.A. Jones and S.F. King, Leptogenesis in the Two Right-Handed Neutrino Model Revisited, Phys. Rev. D 86 (2012) 023516 [arXiv:1107.6002] [InSPIRE].

[65] S. Antusch, P. Di Bari, D.A. Jones and S.F. King, A fuller flavour treatment of $\mathrm{N}_{2}$-dominated leptogenesis, Nucl. Phys. B 856 (2012) 180 [arXiv:1003.5132] [INSPIRE].

[66] R. Samanta, P. Roy and A. Ghosal, Consequences of minimal seesaw with complex $\mu \tau$ antisymmetry of neutrinos, JHEP 06 (2018) 085 [arXiv: 1712.06555] [INSPIRE].

[67] Planck collaboration, Planck intermediate results. XLVI. Reduction of large-scale systematic effects in HFI polarization maps and estimation of the reionization optical depth, Astron. Astrophys. 596 (2016) A107 [arXiv:1605. 02985] [INSPIRE].

[68] I. Esteban, M.C. Gonzalez-Garcia, A. Hernandez-Cabezudo, M. Maltoni and T. Schwetz, Global analysis of three-flavour neutrino oscillations: synergies and tensions in the determination of $\theta_{23}, \delta_{C P}$, and the mass ordering, JHEP 01 (2019) 106 [arXiv:1811.05487] [INSPIRE].

[69] I. Esteban, M.C. Gonzalez-Garcia, M. Maltoni, I. Martinez-Soler and T. Schwetz, Updated fit to three neutrino mixing: exploring the accelerator-reactor complementarity, JHEP 01 (2017) 087 [arXiv: 1611.01514] [INSPIRE].

[70] P. Di Bari, M. Re Fiorentin and R. Samanta, Representing seesaw neutrino models and their motion in lepton flavour space, JHEP 05 (2019) 011 [arXiv: 1812.07720] [INSPIRE].

[71] M.-C. Chen and S.F. King, A4 See-Saw Models and Form Dominance, JHEP 06 (2009) 072 [arXiv:0903.0125] [INSPIRE].

[72] S. Choubey, S.F. King and M. Mitra, On the Vanishing of the CP Asymmetry in Leptogenesis due to Form Dominance, Phys. Rev. D 82 (2010) 033002 [arXiv:1004.3756] [INSPIRE].

[73] G.M. Shore, Faster than light photons in gravitational fields. 2. Dispersion and vacuum polarization, Nucl. Phys. B 633 (2002) 271 [gr-qc/0203034] [INSPIRE].

[74] T.J. Hollowood and G.M. Shore, The refractive index of curved spacetime: The fate of causality in QED, Nucl. Phys. B 795 (2008) 138 [arXiv:0707.2303] [INSPIRE].

[75] T.J. Hollowood and G.M. Shore, The Causal Structure of QED in Curved Spacetime: Analyticity and the Refractive Index, JHEP 12 (2008) 091 [arXiv:0806.1019] [INSPIRE].

[76] G. Engelhard, Y. Grossman, E. Nardi and Y. Nir, The importance of N2 leptogenesis, Phys. Rev. Lett. 99 (2007) 081802 [hep-ph/0612187] [INSPIRE]. 
[77] R. Samanta, A. Biswas and S. Bhattacharya, Non-thermal production of lepton asymmetry and dark matter in minimal seesaw with right handed neutrino induced Higgs potential, arXiv: 2006.02960 [INSPIRE].

[78] S.F. King, Minimal predictive see-saw model with normal neutrino mass hierarchy, JHEP 07 (2013) 137 [arXiv: 1304.6264] [INSPIRE].

[79] P. Di Bari, S. King and M. Re Fiorentin, Strong thermal leptogenesis and the absolute neutrino mass scale, JCAP 03 (2014) 050 [arXiv: 1401.6185] [INSPIRE].

[80] R.Samanta et al., to appear. 